\title{
Regularity issues in the problem of fluid structure interaction
}

\author{
David Gérard-Varet * $\quad$ Matthieu Hillairet ${ }^{\dagger}$
}

October 10, 2018

\begin{abstract}
We investigate the evolution of rigid bodies in a viscous incompressible fluid. The flow is governed by the 2D Navier-Stokes equations, set in a bounded domain with Dirichlet boundary conditions. The boundaries of the solids and the domain have Hölder regularity $C^{1, \alpha}, 0<\alpha \leq 1$. First, we show the existence and uniqueness of strong solutions up to collision. A key ingredient is a BMO bound on the velocity gradient, which substitutes to the standard $H^{2}$ estimate for smoother domains. Then, we study the asymptotic behaviour of one $C^{1, \alpha}$ body falling over a flat surface. We show that collision is possible in finite time if and only if $\alpha<1 / 2$.
\end{abstract}

\section{Introduction}

To understand the dynamics of solid bodies immersed in a fluid is of primary physical interest, with regards to a wide range of phenomena such as sedimentation, filtration, or coagulation. For two-dimensional flows, under the assumption that the $N$ bodies are rigid and homogeneous, and that the fluid is incompressible and viscous, one considers classically the following model:

i) The velocity $u$ and pressure $p$ satisfy Navier-Stokes equations in the fluid domain $F(t)$ :

$$
\begin{aligned}
& \bar{\rho}\left(\partial_{t} u+u \cdot \nabla u\right)-\mu \Delta u=-\nabla p+\bar{\rho} f, \quad x \in F(t) . \\
& \operatorname{div} u=0
\end{aligned}
$$

ii) The $N$ solid bodies are described by the closures $\overline{S^{i}(t)}$ of connected bounded domains $S^{i}(t), 1 \leq i \leq N$. They have rigid velocity fields

$$
u^{i}(t, x)=v^{i}(t)+\omega^{i}(t)\left(x-x^{i}(t)\right)^{\perp}, \quad x \in \overline{S^{i}(t)}, \quad 1 \leq i \leq N,
$$

where $v^{i}$ and $\omega^{i}$ are the translation and angular velocities, whereas $x^{i}$ is the position of the center of mass.

iii) The moving fluid and solid domains occupy a fixed bounded domain $\Omega$ of $\mathbb{R}^{2}$, with Dirichlet boundary condition:

$$
F(t)=\Omega \backslash \cup_{i=1}^{N} \overline{S^{i}(t)}, \quad u=0, \quad x \in \partial \Omega .
$$

${ }^{*}$ DMA/CNRS, Ecole Normale Superieure, 45 rue dUlm, 75005 Paris, France

${ }^{\dagger}$ Université de Toulouse, IMT équipe MIP, 118, route de Narbonne, 31062 Toulouse cedex, France 
iv) The fluid and solid systems are coupled by the continuity of the velocity,

$$
u=u^{i}, \quad x \in \partial S^{i}(t)
$$

and the continuity of the stresses:

$$
\begin{aligned}
& m^{i} \dot{v}^{i}(t)=\int_{\partial S^{i}(t)}\left(\mu \frac{\partial u}{\partial n}-p n\right) d \sigma+\int_{S^{i}(t)} \bar{\rho}^{i} f \\
& J^{i} \dot{\omega}^{i}(t)=\int_{\partial S^{i}(t)}\left(x-x^{i}\right)^{\perp} \cdot\left(\mu \frac{\partial u}{\partial n}-p n\right) d \sigma+\int_{S^{i}(t)}\left(x-x^{i}\right)^{\perp} \cdot \bar{\rho}^{i} f .
\end{aligned}
$$

The positive constants $\bar{\rho}, \mu$ are the density and viscosity of the fluid. The positive constants $m^{i}, J^{i}, \bar{\rho}^{i}$ are the total mass, moment of inertia and density of the $i$-th solid. The source term $f$ models an additional forcing (like gravity). The vector $n$ at the boundary $\partial U$ of an open set $U$ refers as usual to the outward unit normal vector.

Although natural, these equations exhibit some unexpected features, in both two and three dimensions. Hence, consider the case of one rigid body falling in a cavity $(N=1)$, under the action of gravity. It can be shown that if the boundaries of the body and the cavity are smooth, then no collision can occur in finite time. In other words, this system predicts that the kinetic energy of the body is strongly dissipated by the viscosity, resulting in no collision between the body and the boundary. This fact has been known from physicists for many years [4, 6, 9], and was recently proved in one [32] and two dimensions [19, 20].

This no-collision result is of course paradoxical. At the level of medium-sized objects, it goes against Archimede's law, and is clearly denied by common experiments. At a microscopic level, it also lacks relevance, as rebounds between particles are often involved. Many physics papers have been devoted to this paradox, trying to identify the flaw of the previous modelling. We refer to the articles [2, 7] among many. Among the possible explanations, one of the most popular is roughness. Indeed, the no-collision result relies on the fact that the boundary of the solid structure is regular enough (namely $C^{1,1}$ ). Small irregularities could then explain the occurence of collisions, see [26, 28, . Moreover, the effect of surface roughness in the dynamics of particles has been recently emphasized in experiments [8, 23, 33].

The aim of this paper is to study mathematically the roughness-induced effect on the collision process. Therefore, we consider Hölder boundaries. Namely, we assume that

$$
\partial \Omega \in C^{1, \alpha}, \quad \partial S^{i} \in C^{1, \alpha}, \quad \forall i, \quad 0<\alpha \leq 1 .
$$

We will first consider the well-posedness of system (1.1)-(1.5), for such boundaries. We will establish existence and uniqueness of some strong solutions, up to collision. Our result extends previous results obtained for $C^{1,1}$ boundaries. Once this well-posedness is obtained, we will turn to the question of collision in finite time. We will consider the special case of one $C^{1, \alpha}$ rigid body, falling vertically over a horizontal flat surface. Losely, we will show the following:

1. For $\alpha \geq 1 / 2$, no collision can occur, and the strong solution exists for all time.

2. For $\alpha<1 / 2$, one can find solutions for which collision occurs.

This sharp criteria illustrates that roughness might be the reason for collision in fluid structure interaction, and the reason for the apparent paradox of the classical modelling.

Before stating precisely the results, let us mention former mathematical studies. Fluidsolid interaction has been the subject of many papers, mostly devoted to the existence 
theory for problem (1.1)-(1.5). A key ingredient in many existence results is a weak formulation of the equations. Introducing the global quantities

$$
\begin{gathered}
v(t, x):=u(t, x) \mathbf{1}_{F(t)}(x)+\sum_{i=1}^{N} u^{i}(t, x) \mathbf{1}_{S^{i}(t)}(x), \\
\rho(t, x):=\rho^{F}(t, x)+\sum_{i=1}^{N} \rho^{i}(t, x):=\bar{\rho} \mathbf{1}_{F(t)}(x)+\sum_{i=1}^{N} \bar{\rho}^{i} \mathbf{1}_{S^{i}(t)}(x),
\end{gathered}
$$

the conservations of global momentum, global mass, and bodies masses yield respectively: for all $T>0$, for all $\varphi \in \mathcal{V}$, for all $\psi \in \mathcal{D}([0, T) \times \Omega)$,

$$
\begin{aligned}
& \int_{0}^{T} \int_{\Omega}\left(\rho v \cdot \partial_{t} \varphi+\rho v \otimes v: D(\varphi)-2 \mu D(v): D(\varphi)+\rho f \cdot \varphi\right) d x d s+\int_{\Omega} \rho_{0} v_{0} \cdot \varphi(0)=0, \\
& \int_{0}^{T} \int_{\Omega}\left(\rho \partial_{t} \psi+\rho u \cdot \nabla \psi\right)+\int_{\Omega} \rho_{0} \psi(0)=0, \\
& \int_{0}^{T} \int_{\Omega}\left(\rho^{i} \partial_{t} \psi+\rho^{i} u \cdot \nabla \psi\right)+\int_{\Omega} \rho_{0}^{i} \psi(0)=0 .
\end{aligned}
$$

The space of test functions $\mathcal{V}$ is

$$
\mathcal{V}=\left\{\varphi \in \mathcal{D}([0, T) \times \Omega), \quad \nabla \cdot \varphi=0, \quad \rho^{i}(t) D(\varphi)=0, \forall t, \forall 1 \leq i \leq N\right\} .
$$

The divergence, rigidity inside the fluid, and no-slip condition read respectively:

$$
\nabla \cdot v=0, \quad \rho^{i} D(v)=0,1 \leq i \leq N,\left.\quad v\right|_{\partial \Omega}=0 .
$$

We refer to B. Desjardins and M. Esteban [10 for the derivation of these equations. Similarly to $\rho, \rho^{i}$ and $v$, the initial data $\rho_{0}, \rho_{0}^{i}$ and $v_{0}$ are built upon the initial positions of the bodies $S_{0}^{i}$ and the initial fluid and solid velocities $u_{0}, v_{0}^{i}, \omega_{0}^{i}$. We will assume that there is no-contact initially, which means

$$
\overline{S_{0}^{i}} \cap \overline{S_{0}^{j}}=\emptyset, \quad \overline{S_{0}^{i}} \subset \Omega, \quad \forall 1 \leq i, j \leq N, \quad i \neq j .
$$

Broadly speaking, previous studies deal with two kinds of solutions: weak and strong.

Definition $1 A$ weak solution on $(0, T), T>0$, is a family

$$
\left(S^{i}(t), F(t), v\right), \quad 1 \leq i \leq N, \quad F(t)=\Omega \backslash \cup_{i=1}^{N} \overline{S^{i}(t)}
$$

such that

i) $S^{i}(t)$ is a connected bounded domain, for all $0<t<T$, for all $1 \leq i \leq N$.

ii) The scalar functions $\rho, \rho^{i}$ defined in (1.8) and the vector field $v$ satisfy

$$
\left(\rho, \rho^{i}\right) \in L^{\infty}(0, T \times \Omega), \quad v \in L^{\infty}\left(0, T ; L^{2}(\Omega)\right) \cap L^{2}\left(0, T ; H_{0}^{1}(\Omega)\right) .
$$

and equations (1.9), (1.10).

By classical results of R. Di perna and P.-L. Lions [12] on the transport equations $(1.9 \mathrm{~b}, \mathrm{c})$, any $\left(\rho, \rho^{i}, v\right)$ satisfying ii) has the following additional regularity:

$$
\rho, \rho^{i} \in C\left([0, T] ; L^{1}(\Omega)\right),
$$


and the initial data is satisfied in this stronger sense. Moreover, any $\rho^{i}$ satisfying (1.9k) is the characteristic function of a measurable set:

$$
\rho^{i}(t, x)=\mathbf{1}_{S^{i}(t)}(x), \quad \text { for a.e. } t, x
$$

see [25, theorem 2.1, p.23]. However, it is not clear that $S^{i}(t)$ should be open and connected, so that this constraint $i$ ) is added to the definition of a weak solution. Then, using the rigidity condition in (1.10), one can deduce that $v(t, \cdot)$ is a rigid vector field on each $S^{i}(t)$, and by (1.9) , that $S^{i}(t)=\mathcal{R}_{t} S_{0}^{i}$, for a family of affine isometries $\mathcal{R}_{t}$ Lipschitz in $t$.

The existence of global in time $(T=+\infty)$ weak solutions was proved by E. Feireisl [14] and San Martin and coauthors [27] extending earlier studies "up to collision between solids" [11, 21, 22, 5, 18. It holds in dimensions 2 and 3, with initial data satisfying (1.11) and

$$
v_{0} \in L^{2}(\Omega), \quad \operatorname{div} v_{0}=0, \quad f \in L^{2}\left((0, T) ; H^{-1}(\Omega)\right) .
$$

Following the construction by E. Feireisl, no smoothness of the boundaries of the domain and the solids is necessary for the existence of weak solutions. However, the uniqueness of such solutions is unknown in general, even considering dimension 2 and pre-collisional times. After contact, it is known that uniqueness does not hold, as some entropy condition is missing to describe properly the post-collisional dynamics. This suggests to consider stronger solutions, namely

Definition 2 A strong solution on $(0, T)$ is a weak solution with the following additional regularity:

$v \in L^{\infty}\left(0, T ; H_{0}^{1}(\Omega)\right) \cap L^{2}\left(0, T ; W^{1, p}(\Omega)\right)$ for all finite $p, \quad \partial_{t} v \in L^{2}\left(0, T ; L^{2}(\Omega)\right)$.

Our first result is the following:

Theorem 1 (Well-posedness up to collision)

$$
\begin{aligned}
& \text { Let } v_{0} \in H_{0}^{1}(\Omega), \quad \rho_{0}^{i} D\left(v_{0}\right)=0, \forall i, \quad f \in L^{2}\left((0, T) ; W^{1, \infty}(\Omega)\right), \forall T>0 . \text { Assume } \\
& \text { (1.11), and } \partial \Omega \in C^{1, \alpha}, \quad \partial S_{0}^{i} \in C^{1, \alpha}, \quad \forall i, \quad 0<\alpha \leq 1 .
\end{aligned}
$$

Then, there exists a maximal $T_{*} \in(0, \infty]$ with a unique strong solution on $(0, T)$ for all $T<T_{*}$. Moreover, this strong solution exists up to the first collision, which means one of the following alternatives holds true:

i) $T_{*}=\infty, \quad \delta(t)>0 \quad \forall t$.

ii) $T_{*}<\infty, \quad \delta(t)>0 \quad \forall t<T_{*}, \quad \lim _{t \rightarrow T_{*}} \delta(t)=0$,

$$
\text { where } \delta(t):=\min \left\{d\left(S^{i}(t), S^{j}(t)\right), \quad d\left(S^{i}(t), \partial \Omega\right), \quad 1 \leq i, j \leq N, \quad i \neq j\right\} .
$$

Note that by condition (1.11), and the Lipschitz dependance of $\mathcal{R}_{t}$ described above, $\delta$ is positive at least for small times. Our theorem is an extension of results of B. Desjardins and M. Esteban [10, and T. Takahashi [31, who proved respectively existence and uniqueness of strong solutions in the case $\alpha=1$. See also [17] for well-posedness under further technical assumptions on the solids. A key argument in these papers is the classical $L^{2} \mapsto H^{2}$ regularity property for the inverse of the Stokes operator, which holds in $C^{1,1}$ domains. In particular, one can show that

$$
\int_{0}^{T} \int_{F(t)}\left|\nabla^{2} v(t, \cdot)\right|^{2}<+\infty, \quad 0<T<T^{*} .
$$

In the case of general $C^{1, \alpha}$ domains, this $H^{2}$ regularity result is still true away from the boundaries, and $(\rho, v)$ still satisfies (1.1) in the strong sense, that is for almost every 


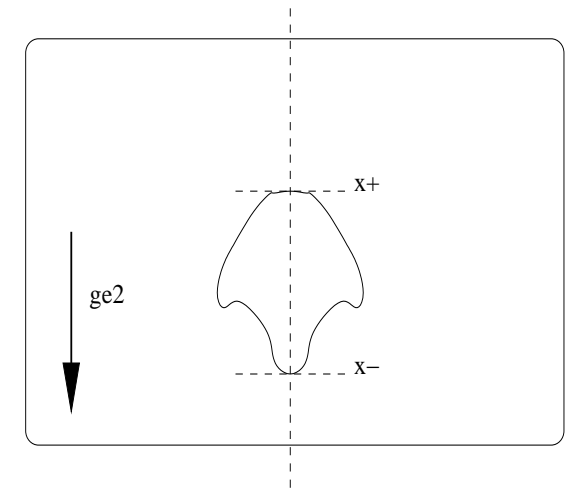

Figure 1: Typical situation

$x, t$. However Theorem 1 requires a control up to the boundary . We will show that the following BMO bound:

$$
\int_{0}^{T}\|\nabla v(t, \cdot)\|_{\mathrm{BMO}(F(t))}^{2}<+\infty, \quad 0<T<T^{*},
$$

substitutes to (1.12), allowing for our well-posedness result.

In a second part, we study if bodies can collide in finite time, that is if $T_{*}$ is finite or not. We consider one $C^{1, \alpha}$ solid that moves vertically near a flat horizontal surface under the action of gravity. More precisely, let us denote $S(t)=\mathcal{R}_{t} S_{0}$ the position of the solid at time $t$. We make the following assumptions:

1. The source term is $f=-g e_{2}$, with $g>0, e_{2}=(0,1)$.

2. The solid moves along the axis $x_{1}=0$, that is $\mathcal{R}_{t}$ is a vertical translation.

3. The only possible collision points are on $x_{1}=0$.

4. Near $x_{1}=0, \partial \Omega$ is flat and horizontal

5. Near $x_{1}=0$, the lower and upper parts of $\partial S(t)$ are given by

$$
x_{2}-x_{-}(t)=\left|x_{1}\right|^{1+\alpha}, \quad x_{2}-x_{+}(t)=-\left|x_{1}\right|^{2}, \quad 0<t<T_{*} .
$$

6. The solid is heavier than the fluid, i.e., $\rho_{\left.\right|_{S(t)}}>\rho_{\left.\right|_{F(t)}}$.

Note that if the initial configuration $\left(\Omega, S_{0}, v_{0}\right)$ is symmetric with respect to the $x_{1}$-axis, then the unique strong solution will be symmetric for all $0<t<T_{*}$, and the solid will move along the vertical axis. Hence, there are plenty of configurations satisfying 1-6. A typical one is shown in figure 1. Our main result is the following:

\section{Theorem 2 (Link between collision and boundary regularity)}

For any strong solution satisfying $1-6, T_{*}<\infty$ if and only if $\alpha<1 / 2$.

In physical terms, the theorem emphasizes the role of roughness in the collision scenario. Our result extends the results of M. Hillairet [20] and T.I. Hesla [19] in the case $\alpha=1$, for which it was shown that no collision occurs. Theorem 2 relies on the study of the stress $\int_{\partial S(t)}\left(\mu \partial_{n} u-p n\right)$. When the boundary is regular, this stress diverges strongly as the distance to the boundary goes to zero. This mechanism prevents collision. When the regularity is weakened, the stress is also weakened, and contact may occur. The proof of the theorem involves the construction of appropriate test functions. In that respect, 
assumptions 2-5 are mostly technical, allowing to handle the computations. As can be seen from our proof, most of our arguments are local, and use only the weak bounds given by the conservation of energy. Hence, we believe that, as far as "real" (not grazing) collisions are concerned, the result might persist for more general domains and weak solutions. However, the source term must remain sufficiently integrable, as shown by an interesting example of Starovoitov [30]. Losely, Starovoitov exhibits an example of a weak solution, colliding in finite time, when $\Omega$ and the solid are two spheres. But the corresponding source term satisfies only

$$
f \in L^{2}\left(0, T ; H^{-1}(\Omega)\right), \quad \forall T>0 .
$$

The $L^{2}$ norm of $f(t, \cdot)$ diverges as $\delta \rightarrow 0, t \rightarrow T_{*}$. This allows to compensate the divergence of the stress and to allow collision, even with regular boundaries. As shown by the first part of our theorem, this phenomenon is ruled out for more realistic forcing (such as gravity).

The rest of the article is organized in three sections. Section 2 gathers regularity properties for the Stokes operator in $C^{1, \alpha}$ domains. Section 3 is devoted to the proof of Theorem 1 . Section 4 contains the proof of Theorem 2 .

\section{Regularity properties in $C^{1, \alpha}$ domains}

Existence and uniqueness of strong solutions have only been considered when solids have $C^{1,1}$ boundaries. More precisely, a key argument in the papers of B. Desjardins and M. Esteban or T. Takahashi is the regularity estimate

$$
\|\nabla u\|_{H^{1}(\mathcal{O})}+\|p\|_{H^{1}(\mathcal{O}) / \mathbb{R}} \leq C\left(\|F\|_{H^{1}(\mathcal{O})}+\|g\|_{H^{1}(\mathcal{O})}\right)
$$

satisfied by the weak solution $(u, p)$ of the Stokes system

$$
\left\{\begin{aligned}
-\Delta u+\nabla p & =\operatorname{div} F, \quad x \in \mathcal{O}, \\
\operatorname{div} u & =g, \quad x \in \mathcal{O}, \\
\left.u\right|_{\partial \mathcal{O}} & =0
\end{aligned}\right.
$$

when $\mathcal{O}$ is a bounded domain with $C^{1,1}$ boundary. Such inequality is no longer valid when $\partial \mathcal{O}$ has weaker regularity. Only the interior estimate

$$
\|\nabla u\|_{H^{1}(K)}+\|p\|_{H^{1}(K) / \mathbb{R}} \leq C(K)\left(\|F\|_{H^{1}(\mathcal{O})}+\|g\|_{H^{1}(\mathcal{O})}\right)
$$

is satisfied, where $K$ is any relatively compact open subset of $\mathcal{O}$. As regards well-posedness issues, this interior bound is not sufficient. We will need a control up to the boundary, given by the following:

Proposition 3 Assume that $\mathcal{O}$ has a $C^{1, \alpha}$ boundary, $0<\alpha \leq 1$. Assume also that

$$
F \in L^{2}(\mathcal{O}) \cap \operatorname{BMO}(\mathcal{O}), \quad g \in L^{2}(\mathcal{O}) \cap \operatorname{BMO}(\mathcal{O}) .
$$

Then, the weak solution $(u, p)$ of (2.2) satisfies

$$
\|(\nabla u, p)\|_{\mathrm{BMO}(\mathcal{O})} \leq C\left(\|(F, g)\|_{\mathrm{BMO}(\mathcal{O})}+\|(F, g)\|_{L^{2}(\mathcal{O})}\right) .
$$

We remind that $\operatorname{BMO}(\mathcal{O})$ is the set of functions $f \in L^{1}(\mathcal{O})$ such that

$$
\sup _{B} \frac{1}{|B|} \int_{B}\left|f(x)-\bar{f}_{B}\right| d x<+\infty, \quad \bar{f}_{B}=\frac{1}{|B|} \int_{B} f(x) d x
$$


where the supremum is taken over all the open balls $B$ of $\mathcal{O}$, that is all the intersections of $\mathcal{O}$ with open disks. Note that the application

$$
\|f\|_{\mathrm{BMO}(\mathcal{O})}:=\sup _{B} \frac{1}{|B|} \int_{B}\left|f(x)-\bar{f}_{B}\right| d x
$$

defines only a semi-norm, as it is invariant by the addition of constants. An easy remark is that $\mathrm{BMO}(\mathcal{O})$ is also characterized by

$$
\sup _{B} \inf _{m}\left(\frac{1}{|B|} \int_{B}|f(x)-m| d x\right)<+\infty,
$$

where the infimum is taken over all real constants, providing an equivalent semi-norm. Note that $f \in \operatorname{BMO}(\mathcal{O})$ if and only if $\tilde{f} \in \operatorname{BMO}\left(\mathbb{R}^{2}\right)$, where $\tilde{f}$ is the extension of $f$ by zero. Hence, standard results for the whole space apply directly to our setting. For instance, $f \in \operatorname{BMO}(\mathcal{O})$ belongs to $L^{p}(\mathcal{O})$ for any finite $p$, and

$$
\sup _{B}\left(\frac{1}{|B|} \int_{B}\left|f(x)-\bar{f}_{B}\right|^{p} d x\right)^{1 / p}<+\infty,
$$

this expression defining again a semi-norm which is equivalent to the previous one. We also remind the Sobolev imbedding in dimension 2:

$$
H^{1}(\mathcal{O}) \hookrightarrow \operatorname{BMO}(\mathcal{O}), \quad\|f\|_{\mathrm{BMO}(\mathcal{O})} \leq C\|f\|_{H^{1}(\mathcal{O})} .
$$

which is simply deduced from Poincaré inequality. Finally, we remind the interpolation inequality: for all $\theta \in(0,1)$, for all $1 \leq p, q<+\infty$ with $(1-\theta) q=p$

$$
\|f\|_{L^{q}(\mathcal{O})} \leq C\|f\|_{L^{p}(\mathcal{O})}^{1-\theta}\|f\|_{\mathrm{BMO}(\mathcal{O})}^{\theta}
$$

We refer to [13, 24] for exhaustive study of the space BMO.

Proof of the proposition. In the case of the whole space $\mathcal{O}=\mathbb{R}^{2}$, the estimate (2.4) follows from the continuity of the Riesz transform on BMO. In the case of a $C^{1, \alpha}$ bounded domain, it is connected to Hölder theory for elliptic systems. Such theory has been of course widely considered, from various perspectives: see [1, 3, 16, 15] for some examples. Although a BMO estimate like (2.4) may be part of the folklore of this domain, we could not find a proper reference for it. For the sake of completeness, we give here the main steps of (one possible) proof. The last step of the proof relies on ideas of Giaquinta et Modica, used to establish Hölder estimates for the Stokes system with Neumann boundary condition [15].

We start with a simple remark, to be used implicitly throughout the sequel: any $f \in L^{2}(U), U$ open set, can be written $f=\operatorname{div} F$, where $F \in H^{1}(U)$ satisfies

$$
\|F\|_{\mathrm{BMO}(U)} \leq C\|F\|_{H^{1}(U)} \leq C^{\prime}\|f\|_{L^{2}(U)}
$$

This will allow to keep the source term in divergence form as we apply transformations to the Stokes system.

Let $(u, p)$ be the weak solution of (2.2), where $p$ is normalized such that $\int_{\mathcal{O}} p=0$. Standard energy estimates yield

$$
\|u\|_{H^{1}(\mathcal{O})}+\|p\|_{L^{2}(\mathcal{O})} \leq C\left(\|F\|_{L^{2}(\mathcal{O})}+\|g\|_{L^{2}(\mathcal{O})}\right) .
$$

Step 1 : Localization. Let $\tilde{\mathcal{O}}^{i} \Subset \mathcal{O}^{i}, i=1 \ldots N$ a covering of $\overline{\mathcal{O}}$ by open sets. Let $\psi^{i}$ a smooth function with compact support in $\mathcal{O}^{i}$, such that $\psi^{i}=1$ on $\tilde{\mathcal{O}}^{i}$. The functions

$$
u^{i}:=\psi^{i} u, \quad p^{i}:=\psi^{i} p
$$


satisfy

$$
\left\{\begin{aligned}
-\Delta u^{i}+\nabla p^{i} & =\operatorname{div}\left(\psi^{i} F\right)-F \nabla \psi^{i}-2(\nabla u)^{t} \nabla \psi^{i}-\Delta \psi^{i} u+p \nabla \psi^{i} \\
& :=\operatorname{div} F^{i}, \quad x \in \mathcal{O}^{i}, \\
\operatorname{div} u & =g \psi^{i}+\nabla \psi^{i} \cdot u:=g^{i}, \quad x \in \mathcal{O}^{i}, \\
\left.u\right|_{\partial \mathcal{O}^{i}} & =0 .
\end{aligned}\right.
$$

By (2.8), the $L^{2} \cap$ BMO norms of $F^{i}$ and $g^{i}$ are controlled by the $L^{2} \cap$ BMO norms of $F$ and $g$. Thus, we can restrict ourselves to a subdomain, that is establish (2.4) with $\mathcal{O}^{i}$ instead of $\mathcal{O}$.

Step 2 : Local coordinates. If $\mathcal{O}^{i}$ does not intersect the boundary of $\mathcal{O}$, the estimate follows from the interior regularity (2.3). If $\mathcal{O}^{i}$ intersects the boundary, we can assume with no loss of generality that it is a local chart: there exists a $C^{1, \alpha}$ diffeomorphism

$$
\chi: \mathcal{O}^{i} \mapsto D(0, R), \quad \chi\left(\mathcal{O}^{i} \cap \partial \mathcal{O}\right)=(-R, R) \times\{0\}, \quad \chi\left(\mathcal{O}^{i} \cap \mathcal{O}\right)=D^{+}(0, R),
$$

where $D^{+}(0, R)$ is the upper half disk of radius $R$ centered at the origin. We define new fields $v, q, F^{\prime}, g^{\prime}$ by the relations

$$
u^{i}(x):=v(\chi(x)), \quad p^{i}(x):=q(\chi(x)), \quad F^{i}(x)=F^{\prime}(\chi(x)), \quad g^{i}(x)=\frac{g^{\prime}(\chi(x))}{\operatorname{det}(\nabla \chi)} .
$$

They satisfy

$$
\left\{\begin{aligned}
-\operatorname{div}(A \nabla v)+\operatorname{div}(B q) & =\operatorname{div}\left(B F^{\prime}\right), & & x \in D^{+}(0, R), \\
B: \nabla v & =g^{\prime}, & & x \in D^{+}(0, R), \\
\left.v\right|_{\partial D^{+}(0, R)} & =0 . & &
\end{aligned}\right.
$$

where

$$
A=\frac{1}{\operatorname{det}(\nabla \chi)}(\nabla \chi)^{t} \nabla \chi, \quad B=\frac{1}{\operatorname{det}(\nabla \chi)}(\nabla \chi)^{t} .
$$

Note that $A$ is uniformly elliptic over $D(0, R)$, and that $A, B$ have $C^{0, \alpha}$ coefficients. As usual, $(\operatorname{div} M)_{i}:=\partial_{j} M_{j i}$, and $M: N=M_{i j} N_{i j}$ for any $2 \mathrm{x} 2$ matrices $M, N$.

Step 3 : Frozen coefficients. We write the previous system as

$$
\left\{\begin{aligned}
-\operatorname{div}(A(0) \nabla v)+\operatorname{div}(B(0) q) & =\operatorname{div}(\tilde{F}), & & x \in D^{+}(0, R), \\
B(0): \nabla v & =\tilde{g}, & & x \in D^{+}(0, R), \\
\left.v\right|_{\partial D^{+}(0, R)} & =0 . & &
\end{aligned}\right.
$$

where

$$
\tilde{F}:=B F^{\prime}-(A(0)-A(x)) \nabla v+(B(0)-B(x)) q, \quad \tilde{g}:=g^{\prime}+(B(0)-B(x)): \nabla v .
$$

Let us assume for a while that $\tilde{F} \in L^{2} \cap \mathrm{BMO}, \tilde{g} \in L^{2} \cap \mathrm{BMO}$, and that the estimate

$$
\|(\nabla v, q)\|_{\mathrm{BMO}\left(D^{+}(0, R)\right)} \leq \mathcal{C}\left(\|(\tilde{F}, \tilde{g})\|_{\mathrm{BMO}\left(D^{+}(0, R)\right)}+\|(\tilde{F}, \tilde{g})\|_{L^{2}\left(D^{+}(0, R)\right)}\right) .
$$

holds. A simple scaling argument shows that the constant $\mathcal{C}$ can be chosen independently of the radius $R$. We now state the following a priori estimate: there exists a universal constant $\mathcal{C}^{\prime}$, and $\varepsilon(R)$ going to zero with $R$ such that

$$
\begin{aligned}
& \|(\tilde{F}, \tilde{g})\|_{\mathrm{BMO}\left(D^{+}(0, R)\right)}+\|(\tilde{F}, \tilde{g})\|_{L^{2}\left(D^{+}(0, R)\right)} \leq \varepsilon(R)\|(\nabla v, q)\|_{\mathrm{BMO}\left(D^{+}(0, R)\right)} \\
& +\mathcal{C}^{\prime}\left(\left\|\left(F^{\prime}, g^{\prime}\right)\right\|_{\mathrm{BMO}\left(D^{+}(0, R)\right)}+\left\|\left(F^{\prime}, g^{\prime}\right)\right\|_{L^{2}\left(D^{+}(0, R)\right)}+\|(\nabla v, q)\|_{L^{2}\left(D^{+}(0, R)\right)}\right)
\end{aligned}
$$


For the sake of brevity, we focus on the BMO bound, as the $L^{2}$ bound is straightforward. More precisely, we just show how to bound $\|(A(0)-A(x)) \nabla v\|_{\text {BMO }}$, because the other terms composing $\tilde{F}$ and $\tilde{g}$ can be handled along the same lines. As emphasized at the beginning of the section, we need to control

$$
I_{B}:=\frac{1}{|B|} \int_{B}|(A(x)-A(0)) \nabla v(x)-c| d x
$$

for any ball $B$ of $D^{+}(0, R)$ and some constant vector $c$ (possibly depending on $B$ ). Let $r$ be the diameter of $B$ and $x_{0}$ a point in $B$. We choose $c=\left(A\left(x_{0}\right)-A(0)\right) \overline{(\nabla v)}_{B}$. We get

$$
\begin{aligned}
I_{B} & \leq \frac{C}{r^{2}}\left(\int_{B}\left|A(x)-A\left(x_{0}\right)\right| \overline{(\nabla v)}_{B} d x+\int_{B}|A(x)-A(0)|\left|\nabla v(x)-\overline{(\nabla v)}_{B}\right| d x\right) \\
& \leq C^{\prime}\left(r^{\alpha-2} \int_{B}|\nabla v(x)| d x+R^{\alpha}\|\nabla v\|_{\mathrm{BMO}\left(D^{+}(0, R)\right)}\right) \\
& \leq C^{\prime}\left(r^{\alpha-2+2 / q}\|\nabla v\|_{L^{p}\left(D^{+}(0, R)\right)}+R^{\alpha}\|\nabla v\|_{\mathrm{BMO}\left(D^{+}(0, R)\right)}\right)
\end{aligned}
$$

for any finite conjugate exponents $p$, q, i.e. $p^{-1}+q^{-1}=1$. We choose $q$ close enough to 1 so that $\alpha-2+2 / q>0$. Together with the interpolation inequality (2.6), we deduce that

$$
\begin{aligned}
I_{B} & \leq C^{\prime \prime}\left(R^{\alpha-2+2 q}\|\nabla v\|_{L^{2}\left(D^{+}(0, R)\right)}^{2 / p}\|\nabla v\|_{\mathrm{BMO}\left(D^{+}(0, R)\right)}^{1-2 / p}+R^{\alpha}\|\nabla v\|_{\mathrm{BMO}\left(D^{+}(0, R)\right)}\right) \\
& \leq C_{1} R^{\gamma}\|\nabla v\|_{\mathrm{BMO}\left(D^{+}(0, R)\right)}+C_{2}\|\nabla v\|_{L^{2}\left(D^{+}(0, R)\right)}
\end{aligned}
$$

for some universal positive constants $\gamma, C_{1}, C_{2}$. The estimate (2.12) follows.

Note that estimates (2.11) and (2.12) yield the bound (2.4). Indeed, up to take smaller $R$, that is up to refine the covering of open sets $\mathcal{O}^{i}$, we can assume that $\varepsilon(R) \leq 1 /(2 \mathcal{C})$. Hence, combining (2.12)-(2.11), we obtain

$$
\begin{aligned}
\frac{1}{2}\|(\nabla v, q)\|_{\mathrm{BMO}\left(D^{+}(0, R)\right)} & \leq \frac{1}{2}\|(\nabla v, q)\|_{L^{2}\left(D^{+}(0, R)\right)} \\
& \left.+\mathcal{C} \mathcal{C}^{\prime}\left(\left\|\left(F^{\prime}, g^{\prime}\right)\right\|_{\mathrm{BMO}\left(D^{+}(0, R)\right)}+\|\left(F^{\prime}, g^{\prime}\right)\right) \|_{L^{2}\left(D^{+}(0, R)\right)}\right) .
\end{aligned}
$$

Then, it is well-known that $L^{2}, H^{1}$ and BMO norms are preserved by $C^{1}$ diffeomorphisms. This allows to bound the right-hand side of the previous inequality:

$$
\begin{aligned}
& \left.\frac{1}{2}\|(\nabla v, q)\|_{L^{2}\left(D^{+}(0, R)\right)}+\mathcal{C C}^{\prime}\left(\left\|\left(F^{\prime}, g^{\prime}\right)\right\|_{\mathrm{BMO}\left(D^{+}(0, R)\right)}+\|\left(F^{\prime}, g^{\prime}\right)\right) \|_{L^{2}\left(D^{+}(0, R)\right)}\right) \\
& \leq C\left(\left\|u^{i}\right\|_{H^{1}\left(\mathcal{O}^{i} \cap \mathcal{O}\right)}+\left\|p^{i}\right\|_{L^{2}\left(\mathcal{O}^{i} \cap \mathcal{O}\right)}+\left\|\left(F^{i}, g^{i}\right)\right\|_{L^{2}\left(\mathcal{O}^{i} \cap \mathcal{O}\right)}+\left\|\left(F^{i}, g^{i}\right)\right\|_{\mathrm{BMO}\left(\mathcal{O}^{i} \cap \mathcal{O}\right)}\right) \\
& \leq C^{\prime}\left(\|(F, g)\|_{L^{2}(\mathcal{O})}+\|(F, g)\|_{\mathrm{BMO}(\mathcal{O})}\right)
\end{aligned}
$$

where the last line involves the basic estimate (2.8). As regards the left-hand side, we obtain the lower bound

$$
\left\|p^{i}\right\|_{\mathrm{BMO}\left(\mathcal{O}^{i} \cap \mathcal{O}\right)} \leq C\|q\|_{\mathrm{BMO}\left(D^{+}(0, R)\right)}
$$

and along the lines of (2.13)

$$
\begin{aligned}
\left\|\nabla u^{i}\right\|_{\mathrm{BMO}\left(\mathcal{O}^{i} \cap \mathcal{O}\right)} & =\|\nabla \chi \nabla v(\chi(\cdot))\|_{\mathrm{BMO}\left(\mathcal{O}^{i} \cap \mathcal{O}\right)}, \\
& \leq C\left(\|\nabla v\|_{\mathrm{BMO}\left(D^{+}(0, R)\right)}+\|\nabla v\|_{L^{2}\left(D^{+}(0, R)\right)}\right) .
\end{aligned}
$$

This altogether implies (2.4).

We stress that (2.11) and (2.12) are only a priori estimates: $\nabla u, p$, and therefore $\nabla v, q$ are only supposed to be in $L^{2}$, and not in BMO. Nevertheless, regularizing the coefficients 
of $A$ and $B$, establishing the same estimates for the regularized problem and passing to the limit allows to show that the weak solutions are indeed in BMO and that the inequality holds. As this regularization argument is very classical, we leave it to the reader.

Step 4: BMO estimate for the Stokes system in a half-disk. The final step of the proof is to derive the estimate (2.11) for the system (2.10). By the reverse change of variables:

$$
x \mapsto\left(\nabla \chi(0)^{t}\right)^{-1} x
$$

we can assume that $A(0)=B(0)=I_{2}$ is the identity matrix. By this linear mapping, the domain $D^{+}(0, R)$ turns into the intersection of a half-plane and an ellipse, say $E^{+}$. As all the vector fields involved are compactly supported in $E^{+}$, this Stokes system with Dirichlet boundary conditions still holds in any half-disk containing $E^{+}$. As this system is rotationally invariant, we can furthermore assume the half-disk to be $D^{+}\left(0, R^{\prime}\right)$ for some large enough $R^{\prime}$. Finally, as the estimate (2.11) is invariant by the dilations $x \mapsto R^{\prime} x$, we can consider the case $R^{\prime}=1$. Eventually, we only have to establish the inequality

$$
\|(\nabla u, p)\|_{\mathrm{BMO}\left(D^{+}(0,1)\right)} \leq C\left(\|(F, g)\|_{\mathrm{BMO}\left(D^{+}(0,1)\right)}+\|(F, g)\|_{L^{2}\left(D^{+}(0,1)\right)}\right)
$$

for the system

$$
\left\{\begin{aligned}
-\Delta u+\nabla p & =\operatorname{div} F, & & x \in D^{+}(0,1), \\
\operatorname{div} u & =g, & & x \in D^{+}(0,1), \\
\left.u\right|_{\partial D^{+}(0,1)} & =0 . & &
\end{aligned}\right.
$$

We remind that, if $p$ is chosen such that $\int_{D^{+}(0,1)} p=0$, we already have the $L^{2}$ estimate

$$
\|(\nabla u, p)\|_{L^{2}\left(D^{+}(0,1)\right)} \leq C\|(F, g)\|_{L^{2}\left(D^{+}(0,1)\right)} .
$$

We shall rely on ideas of Giaquinta and Modica, who prove in article 15] a Hölder estimate for the Stokes equation with Neumann type boundary conditions. Let $0<\rho \leq$ $R \leq 2$, and $y$ in $D^{+}(0,1)$. We will denote

$$
B(y, \rho):=D(y, \rho) \cap D^{+}(0,1), \quad \bar{f}_{y, \rho}:=\frac{1}{|B(y, \rho)|} \int_{B(y, \rho)} f, \quad \forall 0<\rho \leq R .
$$

We decompose $u=v+w, p=q+r$, where $(v, q)$ solves

$$
\left\{\begin{aligned}
-\Delta v+\nabla q & =\operatorname{div} F, & & x \in B(y, R), \\
\operatorname{div} v & =g-\bar{g}_{y, R}, & & x \in B(y, R), \\
\left.v\right|_{\partial B(y, R)} & =0 . & &
\end{aligned}\right.
$$

and $(w, r)$ solves

$$
\left\{\begin{aligned}
-\Delta w+\nabla r & =0, \quad x \in B(y, R), \\
\operatorname{div} w & =\bar{g}_{y, R}, \quad x \in B(y, R), \\
\left.w\right|_{\partial B(y, R)} & =\left.u\right|_{\partial B(y, R)} .
\end{aligned}\right.
$$

We must first derive an estimate on $v$ and $q$. We state without proof the well-known inequality (see [29])

$$
\left\|q-\bar{q}_{y, \rho}\right\|_{L^{2}(B(y, \rho))} \leq C\|\nabla v\|_{L^{2}(B(y, \rho))},
$$

where $C$ does not depend on $\rho$ by a simple scaling argument. Then, a standard energy estimate yields

$$
\int_{B(y, R)}|\nabla v|^{2}=-\int_{B(y, R)}\left(F-\bar{F}_{y, R}\right) \cdot \nabla v+\int_{B(y, R)}\left(g-\bar{g}_{y, R}\right) \cdot\left(q-\bar{q}_{y, R}\right)
$$


which combined with (2.14) yields

$$
\|\nabla v\|_{L^{2}(B(y, R))} \leq C\left(\left\|F-\bar{F}_{y, R}\right\|_{L^{2}(B(y, R))}+\left\|g-\bar{g}_{y, R}\right\|_{L^{2}(B(y, R)}\right) .
$$

We now wish to obtain an estimate on $w$ and $r$. As for $q$, the pressure $r$ satisfies

$$
\left\|r-\bar{r}_{y, \rho}\right\|_{L^{2}(B(y, \rho))} \leq C\|\nabla w\|_{L^{2}(B(y, \rho))},
$$

As regards $w$, we want to show the estimate

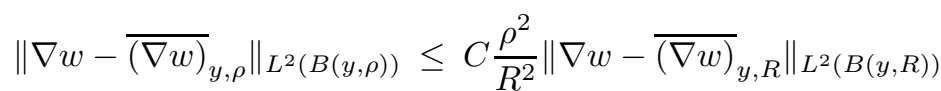

where $C$ does not depend on $\rho$ or $R$. At first, up to replace $w$ by $w-x_{2}\left(\begin{array}{c}{\overline{\left(\partial_{2} w_{1}\right)}}_{y, R} \\ \bar{g}_{y, R}\end{array}\right)$, which would still be zero at the flat part of the boundary $\partial B(y, R) \cap\left\{x_{2}=0\right\}$, and would still satisfy (2.17), we can assume that

$$
{\overline{\left(\partial_{2} w_{1}\right)}}_{y, R}=\bar{g}_{y, R}=0
$$

If $\rho>R / 2$, inequality (2.17) is trivially satisfied. If $\rho<R / 2$, there are two cases.

If $B(y, R) \subset\left\{x_{2}>0\right\}$, the ball $B(y, R)$ does not intersect the boundary of $D^{+}(0,1)$. We can use the interior estimate provided by Giaquinta and Modica in 15: we can apply proposition 1.9, estimate (1.14) to the derivatives of $w$, which are still solutions of the Stokes equation, and this yields exactly (2.17).

$$
\begin{aligned}
& \text { If } B(y, R) \cap\left\{x_{2}=0\right\} \neq \emptyset \text {, we write } \\
& \qquad \begin{aligned}
\left\|\nabla w-\overline{(\nabla w)}_{y, \rho}\right\|_{L^{2}(B(y, \rho))} & \leq C \rho\left\|\nabla^{2} w\right\|_{L^{2}(B(y, \rho))} \\
& \leq C \rho^{2}\left\|\nabla^{2} w\right\|_{L^{\infty}(B(y, \rho))} \leq C \rho^{2}\left\|\nabla^{2} w\right\|_{L^{\infty}(B(y, R / 2))} \\
& \leq C(R) \rho^{2}\|\nabla w\|_{L^{2}(B(y, R))}
\end{aligned}
\end{aligned}
$$

Note that the first inequality is simply Poincaré's inequality, whereas the last one stems from classical regularity results for the Stokes operator. Simple scaling considerations give the bound $C(R) \leq C / R^{2}$ for some constant $C$ that does not depend on $R$. To prove (2.17), it is therefore enough to show that: for any solution $w$ of the Stokes equation

$$
\left\{\begin{aligned}
-\Delta w+\nabla p & =0, & & x \in B(y, R), \\
\operatorname{div} w & =0, & & x \in B(y, R),
\end{aligned}\right.
$$

satisfying moreover

$$
{\overline{\left(\partial_{2} w_{1}\right)}}_{y, R}=0, \quad w=0 \text { on } \quad \partial B(y, R) \cap\left\{x_{2}=0\right\}
$$

we have

$$
\|\nabla w\|_{L^{2}(B(y, R))} \leq C\left\|\nabla w-\overline{(\nabla w)}_{y, R}\right\|_{L^{2}(B(y, R))} \quad \text { if } \partial B(y, R) \cap\left\{x_{2}=0\right\} \neq \emptyset .
$$

Again, the constant $C$ in the r.h.s can be chosen independently of $R$.

If inequality (2.19) were not to be satisfied, one could find solutions $w^{n}$ satisfying (2.18), and such that

$$
\left\|\nabla w^{n}\right\|_{L^{2}(B(y, R))}=1, \quad\left\|\nabla w-{\overline{\left(\nabla w^{n}\right)}}_{y, R}\right\|_{L^{2}(B(y, R))} \underset{n \rightarrow+\infty}{\longrightarrow} 0
$$


From the first equality, up to a subsequence, $w^{n} \rightarrow w$ weakly in $H^{1}(B(y, R))$. This implies the convergence of the averages ${\overline{\left(\nabla w^{n}\right)}}_{y, R} \rightarrow \overline{(\nabla w)}_{y, R}$. Moreover, by standard ellipticity properties of the Stokes operator, we have

$$
\left\|\nabla^{2} w^{n}\right\|_{L^{2}(B)} \leq C(R, B), \quad \forall B \Subset B(y, R)
$$

so that $w^{n} \rightarrow w$ strongly in $H^{1}(B)$. Hence, we obtain, as $n \rightarrow+\infty$,

$$
\nabla w=\overline{(\nabla w)}_{y, R} \quad \text { on } B .
$$

From the second condition in (2.18) and the divergence-free condition, we get

$$
{\overline{\left(\partial_{1} w\right)}}_{y, R}=0, \quad{\overline{\left(\partial_{2} w_{2}\right)_{y, R}}}_{y}=0 .
$$

Moreover, by the first condition in (2.18), we also have ${\overline{\left(\partial_{2} w_{1}\right)}}_{y, R}=0$. Hence, $\overline{(\nabla w)}_{y, R}=$ 0 , and $\nabla w=0$ in any subset $B$ relatively compact in $B(y, R)$. Thus, $\nabla w=0$ on $B(y, R)$ which contradicts the assumption that its $L^{2}$ norm is 1 .

This last argument leads to the desired inequality (2.19) on (2.17). Combining (2.15) and (2.17), we obtain

$$
\begin{aligned}
& \left\|\nabla u-\overline{(\nabla u)}_{y, \rho}\right\|_{L^{2}(B(y, \rho))} \leq\left\|\nabla w-\overline{(\nabla w)}_{y, \rho}\right\|_{L^{2}(B(y, \rho))}+\left\|\nabla v-\overline{(\nabla v)}_{y, \rho}\right\|_{L^{2}(B(y, \rho))} \\
& \leq C\left(\frac{\rho^{2}}{R^{2}}\left\|\nabla w-\overline{(\nabla w)}_{y, R}\right\|_{L^{2}(B(y, R))}+\|\nabla v\|_{L^{2}(B(y, R))}\right) \\
& \leq C^{\prime}\left(\frac{\rho^{2}}{R^{2}}\left\|\nabla u-\overline{(\nabla u)}_{y, R}\right\|_{L^{2}(B(y, R))}+\left\|F-\bar{F}_{y, R}\right\|_{L^{2}(B(y, R))}+\left\|g-\bar{g}_{y, R}\right\|_{L^{2}(B(y, R))}\right) \\
& \leq C^{\prime \prime}\left(\frac{\rho^{2}}{R^{2}}\left\|\nabla u-\overline{(\nabla u)}_{y, R}\right\|_{L^{2}(B(y, R))}+\|(F, g)\|_{\mathrm{BMO}\left(D^{+}(0,1)\right)} R^{2}\right)
\end{aligned}
$$

We use lemma 0.6 of $[15]$ to conclude that

$$
\left\|\nabla u-\overline{(\nabla u)}_{y, \rho}\right\|_{L^{2}(B(y, \rho))} \leq C\|(F, g)\|_{\mathrm{BMO}\left(D^{+}(0,1)\right)} \rho^{2},
$$

which provides the BMO control of $\nabla u$. The BMO control of the pressure $p$ then follows from (2.14), (2.16). This ends the proof.

In the next section, we will use this proposition to show well-posedness of the PDE's system (1.1)-(1.5). Before that, we state a regularity result of Sobolev type for the Stokes system in $C^{1, \alpha}$ domains. It will allow to give a meaning in the trace sense to the stress tensor at the solid boundary $\left.\left(\partial_{n} u-p n\right)\right|_{\partial S^{i}}$.

Proposition 4 Assume that $\mathcal{O}$ has a $C^{1, \alpha}$ boundary, $0<\alpha \leq 1$. Let $s, \tau$ such that $s<\alpha$ and $s \leq 2 / \tau$. Assume that

$$
F \in L^{2}(\mathcal{O}) \cap W^{s, \tau}(\mathcal{O}), \quad g \in L^{2}(\mathcal{O}) \cap W^{s, \tau}(\mathcal{O}) .
$$

Then, the weak solution $(u, p)$ of (2.2) satisfies

$$
\|(\nabla u, p)\|_{W^{s, \tau}(\mathcal{O})} \leq C\left(\|(F, g) v\|_{W^{s, \tau}(\mathcal{O})}+\|(F, g)\|_{L^{2}(\mathcal{O})}\right) .
$$

We remind that for all $0<s<1$, the fractional Sobolev space $W^{s, \tau}(\mathcal{O})$ is the set of measurable functions $u$ such that

$$
\|u\|_{W^{s, \tau}(\mathcal{O})}:=\left(\iint_{\mathcal{O} \times \mathcal{O}} \frac{|u(x)-u(y)|^{\tau}}{|x-y|^{2+s \tau}} d x d y\right)^{1 / \tau}<+\infty .
$$


and this last expression makes it a Banach space. The assumption $s \leq 2 / \tau$ in the proposition ensures the continuous imbedding

$$
H^{1}(\mathcal{O}) \hookrightarrow W^{s, \tau}(\mathcal{O}), \quad\|f\|_{W^{s, \tau}(\mathcal{O})} \leq C\|f\|_{H^{1}(\mathcal{O})} .
$$

Similarly, the constraint $s<\alpha$ is such that $C^{0, \alpha}(\mathcal{O}) \hookrightarrow W^{s, \tau}(\mathcal{O})$.

Sketch of proof of the proposition. The proof of the Sobolev estimate (2.20) mimics the proof of the BMO estimate (2.4), so that we only quote the few changes to be made.

Steps 1 and 2 (localization and use of local coordinates) remain the same, up to the replacement of BMO by $W^{s, \tau}$ in every argument.

In step 3, the only change is in the derivation of

$$
\begin{aligned}
& \|(\tilde{F}, \tilde{g})\|_{W^{s, \tau}\left(D^{+}(0, R)\right)}+\|(\tilde{F}, \tilde{g})\|_{L^{2}\left(D^{+}(0, R)\right)} \leq \varepsilon(R)\|(\nabla v, q)\|_{W^{s, \tau}\left(D^{+}(0, R)\right)} \\
& +\mathcal{C}^{\prime}\left(\left\|\left(F^{\prime}, g^{\prime}\right)\right\|_{W^{s, \tau}\left(D^{+}(0, R)\right)}+\left\|\left(F^{\prime}, g^{\prime}\right)\right\|_{L^{2}\left(D^{+}(0, R)\right)}+\|(\nabla v, q)\|_{L^{2}\left(D^{+}(0, R)\right)}\right)
\end{aligned}
$$

which substitutes to (2.12). Again, we just show how to bound $\|(A(0)-A(x)) \nabla v\|_{W^{s, \tau}}$, as all other terms that compose $\tilde{F}$ and $g$ are treated in the same manner. We write

$$
\begin{aligned}
& \iint_{D^{+}(0, R) \times D^{+}(0, R)} \frac{|(A(0)-A(x)) \nabla v(x)-(A(0)-A(y)) \nabla v(y)|^{\tau}}{|x-y|^{2+s \tau}} d x d y \\
& \leq C\left(\iint_{D^{+}(0, R) \times D^{+}(0, R)}|A(0)-A(x)|^{\tau} \frac{|\nabla v(x)-\nabla v(y)|^{\tau}}{|x-y|^{2+s \tau}} d x d y\right. \\
& \left.\quad+\iint_{D^{+}(0, R) \times D^{+}(0, R)}|\nabla v(y)|^{\tau} \frac{|A(x)-A(y)|^{\tau}}{|x-y|^{2+s \tau}} d x d y\right) \\
& \leq C\left(\|\nabla v\|_{W^{s, \tau}\left(D^{+}(0, R)\right)}^{\tau} \sup _{x \in D^{+}(0, R)}|A(x)-A(0)|^{\tau}+R^{\tau(\alpha-s)}\|\nabla v\|_{L^{\tau}\left(D^{+}(0, R)\right)}^{\tau}\right) \\
& \leq \varepsilon^{\prime}(R)\|\nabla v\|_{W^{s, \tau}\left(D^{+}(0, R)\right)}^{\tau} .
\end{aligned}
$$

which allows to conclude as in the previous proof.

Step 4, that is the $W^{s, \tau}$ estimate, $0<s<1$, for the Stokes equation in a half-disk, follows from a simple interpolation of similar inequalities for $W^{0, \tau}$ and $W^{1, \tau}$.

\section{Strong solutions}

This section is devoted to the proof of theorem 1. Broadly, we shall prove existence and uniqueness of strong solutions as long as the distance between solid boundaries $\delta(t)$ satisfies $\delta(t)>\delta_{0}$, where $\delta_{0}>0$ is arbitrary. The fact that strong solutions can not exist after collision will be discussed eventually. This altogether will of course imply the result. In what follows, constants will depend implicitly on $\delta_{0}$.

We treat separately the existence and uniqueness parts. The existence result follows the lines of 10, whereas the uniqueness result is inspired by 31. We thus rely substantially on these articles, and put the stress only on the changes due to our not so regular $C^{1, \alpha}$ boundaries.

Our (refined) existence result reads:

\section{Proposition 5 (Existence of strong solutions)}

Let $\delta_{0}>0, v_{0} \in H_{0}^{1}(\Omega), \quad \rho_{0}^{i} D\left(v_{0}\right)=0, \forall i, \quad f \in L^{2}((0, T) \times \Omega), \forall T>0$. Assume (1.11), and

$$
\partial \Omega \in C^{1, \alpha}, \quad \partial S_{0}^{i} \in C^{1, \alpha}, \quad \forall i, \quad 0<\alpha \leq 1 .
$$


Then there exists a strong solution on $(0, T)$ for some $T>0$. Moreover, one of the following alternatives holds true:

i) One can take $T$ arbitrarily large and $\delta(t)>\delta_{0}$ for all $t \leq T$.

ii) One can take $T$ such that $\delta(t)>\delta_{0}$ for all $t<T$ and $\lim _{t \rightarrow T} \delta(t)=\delta_{0}$.

In both cases, the strong solution has the additional regularity

$$
\int_{0}^{T}\|\nabla v(t)\|_{\mathrm{BMO}(F(t))}^{2} d t+\int_{0}^{T}\|q\|_{\mathrm{BMO}(F(t))}^{2} d t<+\infty
$$

and

$$
\int_{0}^{T}\|\nabla v(t)\|_{H^{1}\left(F_{\varepsilon}(t)\right)}^{2} d t+\int_{0}^{T}\|q\|_{H^{1}\left(F_{\varepsilon}(t)\right) / \mathbb{R}^{2}}^{2} d t<+\infty,
$$

where $q$ is the corresponding pressure field, and

$$
F_{\varepsilon}(t):=\{x \in F(t) \text { s.t. } \operatorname{dist}(x, \partial F(t))>\varepsilon\}, \quad \varepsilon>0 .
$$

Proof of the proposition. Following [10, we establish a priori estimates for a sufficiently smooth solution $(v, q)$ on $(0, T)$, s.t. $\delta(t)>\delta_{0}$ for all $t<T$.

We first take $\varphi=v$ as a test function, which yields the standard energy inequality

$$
\|v\|_{L^{\infty}\left(0, T ; L^{2}(\Omega)\right)}+\|v\|_{L^{2}\left(0, T ; H_{0}^{1}(\Omega)\right)} \leq C(T)\left(\left\|v_{0}\right\|_{L^{2}(\Omega)}+\|f\|_{L^{2}((0, T) \times \Omega)}\right)
$$

where $C(T)$ is an increasing function of $T$. Then, we take $\varphi=\partial_{t} v$ as a test function, which yields

$$
\int_{0}^{t} \int_{\Omega}\left|\partial_{t} v\right|^{2}+\mu \int_{\Omega}|D(v)(t)|^{2} \leq C\left(\int_{\Omega}\left|D\left(v_{0}\right)\right|^{2}+\int_{0}^{t} \int_{\Omega}|f|^{2}+\int_{0}^{t} \int_{\Omega}|v \cdot \nabla v|^{2}\right)
$$

Note that the l.h.s. in (3.1), resp. (3.2) controls the $L^{\infty} \cap L^{2}$ norm of $v^{i}, \omega^{i}$, resp. the $L^{2}$ norm of $\dot{v}^{i}, \dot{\omega}^{i}$. We now use Stokes regularity to bound the last term in (3.2).

The Navier-Stokes equation for the fluid part can be written

$$
\begin{cases}\mu \Delta v-\nabla q=\bar{\rho}\left(\partial_{t} v+v \cdot \nabla v-f\right), & \operatorname{div} v=0, \quad x \in F(t), \\ \left.v\right|_{\partial S^{i}(t)}=v^{i}(t)+\omega^{i}(t)\left(x-x^{i}(t)\right)^{\perp}, & \left.v\right|_{\partial \Omega \cap \partial F(t)}=0 .\end{cases}
$$

As the solids and the cavity do not touch $\left(\delta(t) \geq \delta_{0}\right)$, it is standard to build a solenoidal vector field $w(t, \cdot) \in H^{\infty}(\Omega)$ such that

$$
\left.w(t, \cdot)\right|_{S^{i}(t)}=v^{i}(t)+\omega^{i}(t)\left(x-x^{i}(t)\right)^{\perp},\left.\quad w(t, \cdot)\right|_{\partial \Omega}=0,
$$

with the estimate $\|w(t, \cdot)\|_{H^{s}} \leq C_{s} \sum_{i}\left(\left|v^{i}(t)\right|+\left|\omega^{i}(t)\right|\right)$. Then, the function $u=v-w$ satisfies

$$
\left\{\begin{array}{l}
\mu \Delta u-\nabla q=\bar{\rho}\left(\partial_{t} v+v \cdot \nabla v-f\right)-\mu \Delta w, \quad \operatorname{div} u=0, \quad x \in F(t), \\
\left.u\right|_{\partial F(t)}=0 .
\end{array}\right.
$$

As $F(t)$ is a $C^{1, \alpha}$ open domain, we can apply the estimates of the previous section. If $q$ is normalized so that $\int_{F(t)} q(t, \cdot)=0$, we have, by (2.7) and propositions 344

$$
\begin{aligned}
\|(\nabla u, q)(t)\|_{L^{2}(F(t))}+ & \|(\nabla u, q)(t)\|_{H^{1}\left(F_{\varepsilon}(t)\right)}+\|(\nabla u, q)(t)\|_{\mathrm{BMO}(F(t))} \\
+\|(\nabla u, q)(t)\|_{W^{s, \tau}(F(t))} \leq & C\left(\left\|\partial_{t} v(t)\right\|_{L^{2}(F(t))}+\|v \cdot \nabla v(t)\|_{L^{2}(F(t))}\right. \\
& \left.+\|f(t)\|_{L^{2}(F(t))}+\sum_{i}\left(\left|v^{i}(t)\right|+\left|\omega^{i}(t)\right|\right)\right) .
\end{aligned}
$$


We remind that this bound holds for all $s, \tau$ such that $s<\alpha$, and $s \leq 2 / \tau$. Back to the original field $v$, and using the interpolation inequality (2.6), we get: for all finite $r$

$$
\begin{aligned}
&\|(\nabla v, q)(t)\|_{L^{r}(F(t))}^{2}+\|(\nabla v, q)(t)\|_{H^{1}\left(F_{\varepsilon}(t)\right)}^{2}+\|(\nabla v, q)(t)\|_{\mathrm{BMO}(F(t))}^{2} \\
&+\|(\nabla v, q)(t)\|_{W^{s, \tau}(F(t))}^{2} \leq C^{\prime}\left(\left\|\partial_{t} v(t)\right\|_{L^{2}(F(t))}^{2}+\|v \cdot \nabla v(t)\|_{L^{2}(F(t))}^{2}\right. \\
&\left.+\|f(t)\|_{L^{2}(F(t))}^{2}+\sum_{i}\left(\left|v^{i}(t)\right|^{2}+\left|\omega^{i}(t)\right|^{2}\right)\right) .
\end{aligned}
$$

By a time integration of (3.4) from 0 to $t$, and a linear combination with (3.1) and (3.2), we obtain

$$
\begin{aligned}
& \int_{0}^{t}\left(\left\|\partial_{t} v(s)\right\|_{L^{2}(\Omega)}^{2}+\|\nabla v(s)\|_{L^{r}(\Omega)}^{2}+\|(\nabla v, q)(s)\|_{H^{1}\left(F_{\varepsilon}(s)\right)}^{2}+\|(\nabla v, q)(s)\right) \|_{\operatorname{BMO}(F(s))}^{2} \\
& \left.+\|(\nabla v, q)(s)\|_{W^{s, \tau}(F(s))}^{2}\right) d s+\|\nabla v(t)\|_{L^{2}(\Omega)}^{2} \\
& \leq C(T)\left(\left\|v_{0}\right\|_{H^{1}(\Omega)}^{2}+\|f\|_{L^{2}((0, T) \times \Omega)}^{2}+\int_{0}^{t}\|v \cdot \nabla v(s)\|_{L^{2}(\Omega)}^{2} d s\right)
\end{aligned}
$$

where $C(T)$ is an increasing function of $T$. To have a closed estimate, it remains to handle the nonlinear term. We split it into

$$
\int_{0}^{t} \int_{\Omega}|v \cdot \nabla v|^{2}=\int_{0}^{t} \int_{F(s)}|v \cdot \nabla v(s)|^{2} d s+\sum_{i} \int_{0}^{t} \int_{S^{i}(s)}|v \cdot \nabla v(s)|^{2} d s
$$

The last term in the decomposition clearly satisfies

$$
\sum_{i} \int_{0}^{t} \int_{S^{i}(s)}|v \cdot \nabla v(s)|^{2} d s \leq C \sum_{i} \int_{0}^{t}\left|v_{i}(s)\right|^{4}+\left|\omega^{i}(s)\right|^{4} d s \leq C^{\prime}
$$

where $C^{\prime}$ depends on $\left\|v_{0}\right\|_{L^{2}}$ and $\|f\|_{L^{2}((0, T) \times \Omega)}$. The first term is bounded in the following way:

$$
\begin{aligned}
\int_{0}^{t}\|v \cdot \nabla v(s)\|_{L^{2}(F(s))}^{2} d s \leq \int_{0}^{t}\|v(s)\|_{L^{4}(F(s))}^{2}\|\nabla v(s)\|_{L^{4}(F(s))}^{2} d s \\
\leq C \int_{0}^{t}\|v(s)\|_{L^{2}(F(s))}\|v(s)\|_{H^{1}(F(s))}\|\nabla v(s)\|_{L^{2}(F(s))}\|\nabla v(s)\|_{\mathrm{BMO}(F(s))} d s \\
\leq C\|v\|_{L^{\infty}\left(0, T ; L^{2}(\Omega)\right)} \int_{0}^{t}\|v(s)\|_{H^{1}(\Omega)}^{2}\|\nabla v(s)\|_{\mathrm{BMO}(F(s))} d s \\
\leq C^{\prime}+\eta \int_{0}^{t}\|\nabla v(s)\|_{\mathrm{BMO}(F(s))}^{2} d s+C_{\eta} \int_{0}^{t}\|\nabla v(s)\|_{L^{2}(\Omega)}^{4}
\end{aligned}
$$

where $\eta$ is arbitrary and $C^{\prime}$ is an increasing function of $\left\|v_{0}\right\|_{L^{2}(\Omega)}$ and $\|f\|_{L^{2}((0, T) \times \Omega)}$. Note that the second line is deduced from the use of Gagliardo-Nirenberg inequality and the interpolation inequality (2.6). Choosing $\eta$ small enough, (3.6) and (3.5) imply that

$$
\|\nabla v(t)\|_{L^{2}(\Omega)} \leq C+\int_{0}^{t}\|\nabla v(s)\|_{L^{2}(\Omega)}^{2}\|\nabla v(s)\|_{L^{2}(\Omega)}^{2} d s .
$$

using Gronwall lemma, and the fact that $\int_{0}^{T}\|\nabla v(s)\|_{H^{1}(\Omega)}^{2}$ is bounded through (3.1), we obtain

$$
\|\nabla v\|_{L^{\infty}\left(0, T, L^{2}(\Omega)\right)} \leq C
$$


where $C$ is an increasing function of $T,\left\|v_{0}\right\|_{H^{1}(\Omega)}$ and $\|f\|_{L^{2}((0, T) \times \Omega)}$. Using this bound in (3.5), we finally obtain:

$$
\begin{aligned}
& \int_{0}^{T}\left(\left\|\partial_{t} v(t)\right\|_{L^{2}(\Omega)}^{2}+\|\nabla v(t)\|_{L^{r}(\Omega)}^{2}+\|(\nabla v, q)(t)\|_{H^{1}\left(F_{\varepsilon}(t)\right)}^{2}+\|(\nabla v, q)(t)\|_{\mathrm{BMO}(F(t))}^{2}\right. \\
& \left.\quad+\|(\nabla v, q)(t)\|_{W^{s, \tau}(F(t))}^{2}\right) d t+\|\nabla v\|_{L^{\infty}\left(0, T, L^{2}(\Omega)\right)} \leq C\left(T,\left\|v_{0}\right\|_{H^{1}(\Omega)},\|f\|_{L^{2}(0, T \times \Omega)}\right) .
\end{aligned}
$$

These a priori estimates are as usual the key element in the construction of strong solutions, as it provides compactness for a sequence of approximate solutions. In the case of $C^{1,1}$ boundaries, the issue of building such approximate solutions and passing to the limit has been adressed in B. Desjardins and M. Esteban, as well as in many other studies. As it adapts straightforwardly to our case, we do not give further detail and refer to these papers.

Let us stress that the $W^{s, \tau}$ regularity of $(\nabla v, q)$ allows to define the stress tensor at the boundary $\left.\left(\partial_{n} v-q n\right)\right|_{\partial F(t)}$. Indeed, taking indices $s, \tau$ such that $\tau s>1$ (together with the requirements $s<\alpha, \quad \tau s \leq 2$ ), one can define the traces of $\nabla v$ and $q$ as elements of $W^{s-1 / \tau, \tau}(\partial F(t))$ for almost all $t$. Note also that the regularity properties

$$
v \in L^{2}\left(0, T ; W^{1,4}(\Omega)\right), \quad \partial_{t} v \in L^{2}\left(0, T ; L^{2}(\Omega)\right)
$$

of a strong solution $v$ are enough to ensure that the right-hand side in (3.3) belongs to $L^{2}((0, T) \times \Omega)$. If $\delta(t) \geq \delta_{0}$ for all $t<T$, this automatically implies the $L^{2}\left(H_{l o c}^{2}\right)$, $L^{2}(\mathrm{BMO})$ and $L^{2}\left(W^{s, \tau}\right)$ bounds on $(\nabla v, q)$ restricted to the fluid domain. This shows the last statement of the proposition, and concludes the existence part.

We now turn to the uniqueness of strong solutions. Our result is

\section{Proposition 6 (Uniqueness of strong solutions)}

Let $\delta_{0}>0, \quad v_{0} \in H_{0}^{1}(\Omega), \quad \rho_{0}^{i} D\left(v_{0}\right)=0, \forall i, \quad f \in L^{2}\left(0, T ; W^{1, \infty}(\Omega)\right), \forall T>0$. Assume (1.11), and

$$
\partial \Omega \in C^{1, \alpha}, \quad \partial S_{0}^{i} \in C^{1, \alpha}, \quad \forall i, \quad 0<\alpha \leq 1 .
$$

There is at most one strong solution on $(0, T)$ such that $\delta(t)>\delta_{0}$ for all $t<T$.

Proof of the proposition. We follow closely the work of T. Takahashi related to $C^{1,1}$ boundaries. We focus on changes due to our not so regular $C^{1, \alpha}$ domains. As in 31, we just consider the case $N=1, f=0$, that is one solid $S(t)$ immersed in the cavity $\Omega$, without forcing. To lighten notations, we also assume that the density $\rho=1$ in the solid and the fluid domains. Minor changes allow to handle the general case.

Step 1: Lagrangian coordinates. The first step in the analysis of uniqueness for this free surface problem is to get back to a fixed domain, by a change of variables of lagrangian type. Let $v_{0} \in H^{1}(\Omega)$ and $S(0)$ the initial velocity field and solid position. We will denote by $h(t)$ the position of the center of mass of the solid at time $t$. We can always assume that $h(0)=0$. Let $(v, q)$ a strong solution on $(0, T)$ such that $\delta(t)>\delta_{0}$ for all $t<T$.

We consider the same change of variables as in [31, paragraph 4.1, p1504]: as $\delta(t)>\delta_{0}$, a solenoidal velocity field $\Lambda(t, x)$ is defined such that

$$
\begin{aligned}
\Lambda(t, x) & =0, & & \text { for } x \text { in an } \delta_{0} / 4 \text { neighborhood of } \partial \Omega, \\
\Lambda(t, x) & =\dot{h}(t)+\omega(t)(x-h(t))^{\perp}, & & \text { for } x \text { in an } \delta_{0} / 4 \text { neighborhood of } S(t) .
\end{aligned}
$$


Then, one considers the flow

$$
X(t, \cdot): \Omega \rightarrow \Omega, \quad \frac{\partial}{\partial t} X(y, t)=\Lambda(t, X(t, y)), \quad X(0, y)=y .
$$

which maps $S(0)$ to $S(t)$ and $F(0)$ to $F(t)$. More precisely, in a neighborhood of $S(0)$,

$$
X(t, y)=h(t)+R_{\theta(t)} y, \quad \theta(t)=\int_{0}^{t} \omega(s) d s, \quad R_{\theta}=\left(\begin{array}{cc}
\cos \theta-\sin \theta \\
\sin \theta & \cos \theta
\end{array}\right) .
$$

and near $\partial \Omega, X(t, y)=y$. Note that, as $(v, q)$ is a strong solution, $h, \theta \in H^{2}(0, T)$. The mapping $X$ inherits the regularity estimate

$$
\left\|\partial_{t}^{i} X(t, \cdot)\right\|_{H^{s}(\Omega)} \leq C_{s}\left(\left|h^{(i)}(t)\right|+\left|\theta^{(i)}(t)\right|\right), \quad \forall i=0,1,2, \quad \forall s \in \mathbb{N} .
$$

We then introduce the new functions

$$
u(t, y):=(\nabla Y)^{t}(t, X(t, y)) v(t, X(t, y)), \quad p(t, y):=q(t, X(t, y)) .
$$

where $Y:=X^{-1}$ denotes the inverse of $X$ with respect to the space variable, and as usual $(\nabla Y)_{i j}=\partial_{x_{i}} Y_{j}$.

Following [31, paragraph 4.2, p1507], equations (1.1)-(1.5) turn into

$$
\left\{\begin{array}{l}
\partial_{t} u+M u+N u-\mu L u+G p=0, \quad y \in F(0), \\
\operatorname{div} u=0, \quad y \in F(0), \\
u(y, t)=R_{-\theta(t)} \dot{h}(t)+\omega(t) y^{\perp}, \quad y \in \overline{S(0)} \\
m \ddot{h}(t)=R_{\theta(t)} \int_{\partial S(0)}(\mu \nabla u-p) n d y \\
J \dot{\omega}(t)=\int_{\partial S(0)}(\mu \nabla u-p) n \cdot y^{\perp} d y,
\end{array}\right.
$$

plus the initial condition

$$
\left.u\right|_{t=0}(y)=u_{0}(t, y):=v_{0}(t, X(t, y)) .
$$

We refer to 31 for the exact expression of the various operators. In short, $\left(\partial_{t}+M\right) u$ corresponds to the original time derivative $\partial_{t} v, N u$ corresponds to $v \cdot \nabla v, L u$ corresponds to $\Delta v$, and $G p$ corresponds to $\nabla p$. An important point is that

$$
N u=u \cdot \nabla u, \quad L u=\Delta u, \quad G p=\nabla p \quad \text { near } \partial \Omega \text { and } S(0) .
$$

Indeed, we have $X(t, y)=y$ near $\partial \Omega$, so that the change of variables is trivial near the boundary of the cavity. Similarly, $\nabla X(t, y)=R_{\theta(t)}$ near $S(0)$. As Navier-Stokes equations are rotationally invariant, we get (3.8).

Step 2: Stokes like formulation. The operators above involve the flow $X(t, \cdot)$, which depends on the solution $u$ itself: hence, they are nonlinear. But $X(0, y)=y$, which means that for small time, nonlinearities are expected to be small. We shall therefore treat these nonlinear perturbations as source terms. We introduce

$$
H(t):=\int_{0}^{t} R_{-\theta(s)} \dot{h}(s) d s
$$


and write the system as:

$$
\left\{\begin{array}{l}
\partial_{t} u-\mu \Delta u+\nabla p=f-M u-u \cdot \nabla u, \quad y \in F(0) \\
\operatorname{div} u=0, \quad y \in F(0) \\
u(y, t)=\dot{H}(t)+\omega(t) y^{\perp}, \quad y \in \overline{S(0)} \\
m \ddot{H}(t)=\int_{\partial S(0)}(\mu \nabla u-p) n d y+w(t) \\
J \dot{\omega}(t)=\int_{\partial S(0)}(\mu \nabla u-p) n \cdot y^{\perp} d y
\end{array}\right.
$$

where

$$
f:=-(N u-u \cdot \nabla u)+\mu(L-\Delta) u-(G-\nabla) p,
$$

and

$$
w(t)=m \omega(t) R_{\theta(t)} \dot{h}(t)^{\perp} .
$$

Step 3: Uniqueness. The uniqueness of the strong solution will be established thanks to the formulation (3.9). Let $\left(v^{1}, q^{1}\right),\left(v^{2}, q^{2}\right)$ two strong solutions on $(0, T), T>0$, corresponding to the same initial velocity field $v_{0} \in H^{1}(\Omega)$ and same initial configuration $S(0), F(0)=\Omega \backslash \overline{S(0)}$. We remind that for the sake of brevity, we consider the force-free case. We assume that the boundaries $\partial \Omega$ and $\partial S(0)$ are $C^{1, \alpha}$, and that

$$
\delta^{1}(t)>\delta_{0}, \quad \delta^{2}(t)>\delta_{0}, \quad \forall t \in[0, T) .
$$

We can associate to $v^{i}$ the change of variable $X^{i}$, the new functions $u^{i}, p^{i}$ and so on. We shall prove that $u^{1}=u^{2}$ on $(0, T) \times \Omega$. The differences

$$
u:=u^{1}-u^{2}, \quad H:=H^{1}-H^{2}, \quad \omega:=\omega^{1}-\omega^{2}
$$

satisfy with obvious notations

$$
\left\{\begin{array}{l}
\partial_{t} u-\mu \Delta u+\nabla p=f^{1}-f^{2}+M^{2} u^{2}-M^{1} u^{1}+u^{2} \cdot \nabla u^{2}-u^{1} \cdot \nabla u^{1}, \quad y \in F(0) \\
\operatorname{div} u=0, \quad y \in F(0), \\
u(y, t)=\dot{H}(t)+\omega(t) y^{\perp}, \quad y \in \overline{S(0)} \\
m \dot{H}(t)=\int_{\partial S(0)}(\mu \nabla u-p) n d y+w^{1}(t)-w^{2}(t) \\
J \dot{\omega}(t)=\int_{\partial S(0)}(\mu \nabla u-p) n \cdot y^{\perp} d y
\end{array}\right.
$$

with initial condition $\left.u\right|_{t=0}=0$. Now, we can perform the exact same estimates as those performed earlier to show existence of strong solutions. In particular, we get (see estimate (3.5)

$$
\begin{aligned}
\int_{0}^{T} & \left(\left\|\partial_{t} u(t)\right\|_{L^{2}(\Omega)}^{2}+\|\nabla u(t)\|_{L^{r}(\Omega)}^{2}+\|\nabla u(t)\|_{H^{1}\left(F_{\varepsilon}(0)\right)}^{2}+\|\nabla u(t)\|_{\mathrm{BMO}(F(0))}^{2}\right. \\
& \left.+\|p(t)\|_{H^{1}\left(F_{\varepsilon}(0)\right)}^{2}\right) d t+\|\nabla u\|_{L^{\infty}\left(0, T ; L^{2}(\Omega)\right)} \\
& \leq \mathrm{RHS}:=C(T)\left(\left\|f^{1}-f^{2}\right\|_{L^{2}((0, T) \times \Omega)}^{2}+\left\|u^{1} \cdot \nabla u^{1}-u^{2} \cdot \nabla u^{2}\right\|_{L^{2}((0, T) \times \Omega)}^{2}\right. \\
& \left.+\left\|M^{1} u^{1}-M^{2} u^{2}\right\|_{L^{2}((0, T) \times \Omega)}^{2}+\left\|w^{1}-w^{2}\right\|_{L^{2}(0, T)}\right)
\end{aligned}
$$

where $\varepsilon$ is any constant lower than $\delta_{0} / 4$, and $C(T)$ an increasing function of $T$. As usual, the pressure $p$ is normalized so that $\int_{F(0)} p=0$. It remains to estimate the right-hand side. 
By the remark (3.8), $f^{1}-f^{2}$ has a support $F_{\varepsilon}$ which is compact in $F(0)$. The $L^{2}\left(H^{1}\right)$ bound on $(\nabla u, p)$, which was true up to the boundary for $C^{1,1}$ domains, holds in $F_{\varepsilon}$. Moreover, the $L^{2}((0, T) \times \Omega)$ estimate on $\partial_{t} u$ and the $L^{\infty}\left(0, T ; L^{2}(\Omega)\right)$ estimate on $\nabla u$ also hold. Hence, the same bounds as those derived in [31, Corollary 6.16, p1523] apply:

$$
\begin{aligned}
\left\|f^{1}-f^{2}\right\|_{L^{2}(0, T \times \Omega)} \leq \mathcal{C} T^{1 / 10} & \left(\|(\nabla u, p)\|_{L^{2}\left(0, T ; H^{1}\left(F_{\varepsilon}(0)\right)\right.}\right. \\
+ & \left.\left\|\partial_{t} u\right\|_{L^{2}((0, T) \times \Omega)}+\|\nabla u\|_{L^{\infty}\left(0, T ; L^{2}(\Omega)\right)}\right)
\end{aligned}
$$

where $\mathcal{C}$ denotes here and in the sequel an increasing function of $T$ and $\left\|v_{0}\right\|_{H^{1}(\Omega)}$.

As the $L^{2}$ bound on $\partial_{t} u$ is still available, we deduce as in [31, corollary 6.16], that

$$
\left\|w^{1}-w^{2}\right\|_{L^{2}\left(H^{2}\right)} \leq \mathcal{C} T^{1 / 2}\left\|\partial_{t} u\right\|_{L^{2}((0, T) \times \Omega)} .
$$

We remind that $M u=\omega(t) u^{\perp}+\partial_{t} Y \cdot \nabla u$, . Therefore,

$$
\begin{aligned}
& \left\|M^{1} u^{1}-M^{2} u^{2}\right\|_{L^{2}((0, T) \times \Omega)} \leq\left\|M^{1} u\right\|_{L^{2}((0, T) \times \Omega)}+\left\|M u^{2}\right\|_{L^{2}((0, T) \times \Omega)} \\
& \leq C\left(\left\|\left(\omega^{1}, \dot{h}^{1}\right)\right\|_{L^{2}(0, T)}\|u\|_{L^{2}\left(0, T ; H^{1}\right)}+\|(\omega, \dot{h})\|_{L^{2}(0, T)}\left\|u^{2}\right\|_{L^{2}\left(0, T ; H^{1}\right)}\right) \\
& \leq \mathcal{C} T^{1 / 2}\|\nabla u\|_{L^{\infty}\left(0, T ; H^{1}(\Omega)\right)} .
\end{aligned}
$$

Finally, we must control the quadratic term

$$
u^{1} \cdot \nabla u^{1}-u^{2} \cdot \nabla u^{2}=u \cdot \nabla u^{1}+u^{2} \cdot \nabla u .
$$

Like in previous computations, we get

$$
\begin{aligned}
\left\|u \cdot \nabla u^{1}\right\|_{L^{2}((0, T) \times F(0))}^{2} & \leq C\|u\|_{L^{\infty}\left(0, T ; L^{4}(\Omega)\right)}^{2}\left\|\nabla u^{1}\right\|_{L^{\infty}\left(L^{2}(\Omega)\right)} \int_{0}^{T}\left\|\nabla u^{1}(t)\right\|_{\mathrm{BMO}(F(0))} \\
& \leq \mathcal{C} \sqrt{T}\|\nabla u\|_{L^{\infty}\left(0, T ; H^{1}(\Omega)\right)}^{2}
\end{aligned}
$$

using the $L^{2}(\mathrm{BMO})$ bound on $\nabla u^{1}$. Similarly,

$$
\begin{aligned}
\left\|u^{2} \cdot \nabla u\right\|_{L^{2}((0, T) \times F(0))}^{2} & \leq C\left\|u^{2}\right\|_{L^{\infty}\left(L^{4}(\Omega)\right)}^{2}\|\nabla u\|_{L^{\infty}\left(L^{2}(\Omega)\right)} \int_{0}^{T}\|\nabla u(t)\|_{\operatorname{BMO}(F(0))} \\
& \leq \mathcal{C} \sqrt{T}\left(\|u\|_{L^{\infty}\left(H^{1}(\Omega)\right)}^{2}+\|\nabla u\|_{L^{2}(\operatorname{BMO}(F(0)))}\right) .
\end{aligned}
$$

The $L^{2}$ bound in $S(0)$ is straightforward, and we end up with

$$
\left\|u^{1} \cdot \nabla u^{1}-u^{2} \cdot \nabla u^{2}\right\|_{L^{2}(0, T \times \Omega)} \leq \mathcal{C} T^{1 / 4}\left(\|u\|_{L^{\infty}\left(H^{1}(\Omega)\right)}^{2}+\|\nabla u\|_{L^{2}(\operatorname{BMO}(F(0)))}\right)
$$

Eventually, these inequalities lead to

$$
\begin{aligned}
\mathrm{RHS} \leq \mathcal{C} T^{1 / 10} & \left(\|u\|_{L^{\infty}\left(H^{1}(\Omega)\right)}+\left\|\partial_{t} u\right\|_{L^{2}\left(L^{2}(\Omega)\right)}\right. \\
& \left.+\|\nabla u\|_{L^{2}(\operatorname{BMO}(F(0)))}+\|(\nabla u, p)\|_{L^{2}\left(H^{1}\left(F_{\varepsilon}\right)\right)}\right)
\end{aligned}
$$

with $\mathcal{C}$ an increasing function of $T$ and $\left\|v_{0}\right\|_{H^{1}(\Omega)}$. By reporting this bound in (3.11), we deduce that there exists a small $T_{0}$ such that $u^{1}=u^{2}$ on $\left[0, T_{0}\right]$. Moreover, $T_{0}$ depends only on $\left\|v_{0}\right\|_{H^{1}(\Omega)}$ (decreasing as $\left\|v_{0}\right\|_{H^{1}(\Omega)}$ increases). 
Global uniqueness follows. Indeed, we know that $u^{1}$ and $u^{2}$ are in $L^{\infty}\left([0, T], H^{1}(\Omega)\right)$ for all times $T$ such that $\forall t \leq T, \delta^{1}(t), \delta^{2}(t) \geq \delta_{0}$. Hence, up to consider a smaller $T_{0}$, we can apply the above local uniqueness result on $\left[T_{0}, 2 T_{0}\right]$, then $\left[2 T_{0}, 3 T_{0}\right]$ and so on up to reach time $T$. This concludes the uniqueness part.

So far, we have shown existence and uniqueness of strong solutions at least up to the first collision. Theorem 1 asserts more, namely that no strong solution can exist beyond the first collision time. This result can be deduced from 30, Theorems 3.1 and 3.2b]. Indeed, V. Starovoitov has shown the following: suppose that two $C^{1, \alpha}$ solids $S^{i_{1}}(t)$ and $S^{i_{2}}(t)$, resp. a $C^{1, \alpha}$ solid $S^{i}(t)$ and the $C^{1, \alpha}$ cavity $\Omega$, collide for the first time at $t=T$. Denote for $t \leq T$,

$$
\left.h_{i_{1}, i_{2}}(t):=\operatorname{dist}\left(S^{i_{1}}(t), S^{i_{2}}(t)\right)\right) \quad \text { resp. } h_{i}(t):=\operatorname{dist}\left(S^{i}(t), \Omega\right)
$$

and assume that

$$
u \in L^{\infty}\left(0, T ; L^{2}(\Omega)\right) \cap L^{1}\left(0, T ; W^{1, p}(\Omega)\right) .
$$

Then, $h_{i}, h_{i_{1}, i_{2}}$ are lipschitz continuous on $[0, T]$ and, for example,

$$
\left|\frac{d h_{i}}{d t}(t)\right| \leq C h_{i}(t)^{\beta}\|u(t)\|_{W^{1, p}(\Omega)}, \quad \beta=2-\frac{1}{1+\alpha} \frac{p+1}{p}-\frac{1}{p},
$$

for almost all $t \leq T$.

In particular, if $T$ is the first collision time, and the strong solution exists beyond it, the regularity assumption (3.12) is satisfied for arbitrary $p$. Taking $p$ large enough, one can assume that $\beta \geq 1$. We note also that, by hypothesis (1.11), $h(0) \neq 0$. Then, by integration of the previous differential inequality, we obtain $h(T) \neq 0$, which yields a contradiction.

We emphasize that for strong solutions, (3.12) holds a priori only for $T<T_{*}$. Thus, the argument of Starovoitov does not allow to conclude on the occurence of collision. In the next section, we will exhibit configurations for which collision occurs. For such examples, we have:

$$
\int_{0}^{T_{*}}\|u\|_{W^{1, p}(\Omega)}=\infty
$$

\section{The collision/no-collision result}

This section is devoted to the proof of Theorem 2. We consider the simplified configuration described at the end of the introduction, assumptions 1-6. In this framework, the position of the solid is characterized by $h(t):=\operatorname{dist}\left(\left(0, x_{-}(t)\right), \partial \Omega\right)$. Later on, it will be convenient to use a parametrization by $h$, i.e. the translated domains

$$
S_{h}:=S(0)+(h-h(0)) e_{2}, \quad h \in \mathbb{R} .
$$

Of course, $S_{h(t)}=S(t)$. By assumption 5 , the boundary of $S(t)$ is $C^{1,1}$ near its "upper tip" $\left(0, x_{+}(t)\right)$, so that contact is impossible at this point, $c f$. [20. By assumption 6, gravity pushes $S(t)$ downwards. So, we can even assume that

$$
\inf _{t \in\left(0, T_{*}\right)} \operatorname{dist}\left(\left(0, x_{+}(t)\right), \partial \Omega\right)>0 .
$$

Thus, collision can occur if and only if $\lim _{t \rightarrow T_{*}} h(t)=0$. We will show that it is equivalent to $\alpha<1 / 2$. The proof is based on the use of a quasistationary velocity field $w$ and quasistationary pressure field $q$. By quasistationary, we mean that for all $t<T_{*}$,

$$
w(t, x)=w_{h(t)}(x), \quad q(t, x)=q_{h(t)}(x)
$$


for some stationary fields $w_{h}(\cdot), p_{h}(\cdot)$ defined on $\Omega$ and parametrized by $h>0$. Moreover, they will satisfy

$$
\begin{gathered}
w \in C^{1}\left(\left[0, T_{*}\right) ; H^{1}(\Omega)\right), \quad \Delta w(t, \cdot) \in L^{p}(F(t)), p \text { small enough, } t \in\left(0, T_{*}\right), \\
\operatorname{div} w=0 \text { in } \Omega,\left.\quad w\right|_{S(t)}=e_{2},\left.\quad w\right|_{\partial \Omega}=0 .
\end{gathered}
$$

as well as

$$
q \in C^{1}\left(\left[0, T_{*}\right), L^{2}(\Omega)\right), \quad \nabla q(t, \cdot) \in L^{p}(F(t)), p \text { small enough, } t \in\left(0, T_{*}\right) .
$$

In particular, we can use $w$ as a test function in the variational formulation to get

$$
\begin{aligned}
& \int_{0}^{t} \int_{\Omega}\left(\rho v \cdot \partial_{t} w+\rho v \otimes v: D(w)-2 \mu D(v): D(w)-\rho g e_{2} \cdot w\right) \\
& =\int_{\Omega} \rho(t) v(t) \cdot w(t)-\int_{\Omega} \rho(0) v_{0} \cdot w(0)
\end{aligned}
$$

Note that by (4.3)

$$
\begin{aligned}
\int_{\Omega} \rho g e_{2} \cdot w & =\int_{S(t)} \rho g e_{2} \cdot e_{2}+\int_{F(t)} \rho g \nabla\left(x \mapsto x_{2}\right) \cdot w \\
& =\rho_{S} g|S(0)|+\rho_{F} g \int_{\partial F(t)} x_{2} e_{2} \cdot n=\left(\rho_{S}-\rho_{F}\right) g|S(0)|
\end{aligned}
$$

where $\rho_{S}:=\left.\rho\right|_{S(t)}, \rho_{F}:=\left.\rho\right|_{F(t)}$. We also write

$$
\begin{aligned}
2 \mu \int_{\Omega} D(v)(t): D(w)(t) & =\dot{h}(t) \int_{\partial F(t)}\left(\mu \frac{\partial w}{\partial n}-q n\right) \cdot e_{2}-\int_{F(t)}(\mu \Delta w-\nabla q) v \\
& :=\dot{h}(t) n(h)-\int_{F(t)}(\mu \Delta w-\nabla q) \cdot v
\end{aligned}
$$

Thus, the variational formulation yields

$$
N(h(t))+\left(\rho_{S}-\rho_{F}\right) g|S(0)| t=R(t)
$$

where $N$ is the antiderivative of $n$ that vanishes at $h(0)$, and the remainder is

$$
\begin{aligned}
R(t) & :=\int_{0}^{t} \int_{\Omega}\left(\rho v \cdot \partial_{t} w+\rho v \otimes v: D(w)\right)+\int_{\Omega} \rho(0) v_{0} \cdot w(0)-\int_{\Omega} \rho(t) v(t) \cdot w(t) \\
& +\int_{0}^{t} \int_{F(s)} \Delta w(s, \cdot)-\nabla q(s, \cdot) \cdot v(s, \cdot) .
\end{aligned}
$$

Theorem 2 will be deduced from the following proposition:

Proposition 7 One can find $w_{h}: \Omega \mapsto \mathbb{R}^{2}, q_{h}: \Omega \mapsto \mathbb{R}$, such that $w, q$ satisfy (4.2), (4.3), (4.4), and such that

i) For $h>0$ small enough

$$
-c \leq n(h) \leq C h^{-\beta}, \quad \beta=\frac{3 \alpha}{1+\alpha}, \quad c, C>0 .
$$

ii) For all $t<T_{*}$,

$$
|R(t)| \leq C\left(\left\|u_{0}\right\|_{L^{2}}\right)(1+\sqrt{t})
$$


Before tackling the proof of this proposition, let us show how it implies Theorem 2 .

If $\alpha \geq 1 / 2$, then $\beta \geq 1$. We get from (4.5) and point ii) of the proposition:

$$
N(h(t)) \geq\left(\rho_{F}-\rho_{S}\right)|S(0)| t-C(1+\sqrt{t})
$$

By point i), we also get for $h$ small enough

$$
N(h) \leq-C|\ln (h)|
$$

In fact, one can take $h^{1-\beta}$ instead of $|\ln (h)|$ when $\beta>1$, i.e. $\alpha>1 / 2$. Combining those inequalities, we deduce

$$
C|\ln h(t)| \leq\left(\rho_{S}-\rho_{F}\right)|S(0)| t+C(1+\sqrt{t})<+\infty, \quad \forall t<T_{*}
$$

which means that $h$ does not go to zero in finite time. Hence, $T_{*}=+\infty$ and there is no collision

If $\alpha<1 / 2$, then $\beta<1$, and $n \in L^{1}$. Thus, $N$ is continuous. As $h(t)$ is bounded, we deduce from (4.5): $\forall t<T_{*} \leq+\infty$,

$$
-\infty<\inf _{t \in\left(0, T_{*}\right)} N(h(t)) \leq\left(\rho_{F}-\rho_{S}\right)|S(0)| t+C(1+\sqrt{t}) .
$$

If $T_{*}=+\infty$, and $\rho_{S}>\rho_{F}$, one can let $t \rightarrow+\infty$ in the previous inequality. As the r.h.s. goes to $-\infty$ in this limit, it yields a contradiction. Thus, $T_{*}<+\infty$. This ends the proof.

The rest of the paper will be devoted to the proof of Proposition 7 .

\subsection{Construction of the test function}

We mimic the construction presented in article 20] for $C^{1,1}$ boundaries. We want a function $w_{h}(x)$ such that

$$
\operatorname{div} w_{h}=0 \text { in } \Omega,\left.\quad w_{h}\right|_{S_{h}}=e_{2},\left.\quad w_{h}\right|_{\partial \Omega}=0 .
$$

We always consider $0<h<h_{M}:=\sup _{0<t<T_{*}} h(t)$, as no other value of $h$ is involved in our problem.

By a change of coordinates, we can assume $(0,0) \in \partial \Omega$, i.e. $x_{-}(t)=h(t)$. By assumption 5 , there exists $\delta>0$, such that

$$
\forall x \in \partial S_{h} \cap D((0, h), 2 \delta), \quad x_{2}=\gamma_{h}\left(x_{1}\right):=h+\left|x_{1}\right|^{1+\alpha},
$$

where as usual $D(x, r)$ is the disk of center $x$ and radius $r$. Moreover, by assumption 3 and (4.1),

$$
\delta_{\min }:=\inf _{0, h<h_{M}} \operatorname{dist}\left(\partial S_{h} \cap D((0, h), \delta)^{c}, \partial \Omega\right)>0 .
$$

To describe $w_{h}$ away from the origin, we introduce a smooth function $\varphi=\varphi(x), x \in \mathbb{R}^{2}$ such that

$\varphi=1$ in a $\delta_{\min } / 2$-neighborhood of $S_{h(0)}, \quad \varphi=0$ outside a $\delta_{m i n}$-neighborhood of $S_{h(0)}$,

We introduce another smooth function $\chi=\chi(x), x \in \mathbb{R}^{2}$, such that

$$
\chi=1 \text { in }(-\delta, \delta)^{2}, \quad \chi=0 \text { outside }(-2 \delta, 2 \delta)^{2} .
$$

Finally, we set $w_{h}=\nabla^{\perp}\left(x_{1} \varphi_{h}\right)$, with

$$
\begin{aligned}
& \varphi_{h}=1 \quad \text { in } S_{h}, \\
& \varphi_{h}=(1-\chi(x)) \varphi\left(x_{1}, x_{2}-h+h(0)\right)+\chi(x) \frac{x_{2}^{2}}{\gamma_{h}\left(x_{1}\right)^{2}}\left(3-\frac{2 x_{2}}{\gamma_{h}\left(x_{1}\right)}\right) \quad \text { in } \Omega \backslash S_{h} .
\end{aligned}
$$




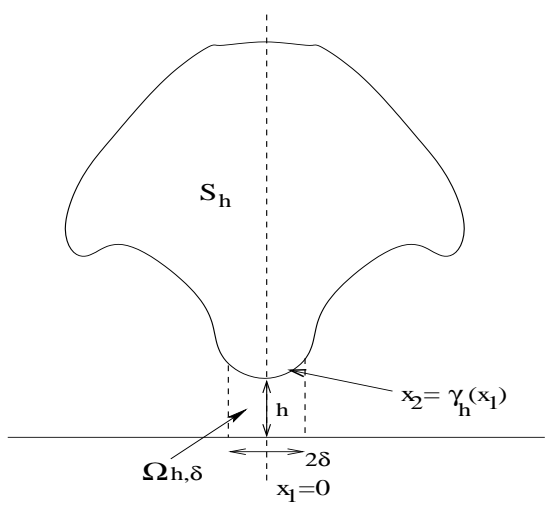

Figure 2: Geometry of the possible contact zone

See figure 4.1 to clarify the main notations. Note that $\varphi_{h}$ and therefore $w_{h}$ are regular up to $h=0$ outside

$$
\Omega_{h, \delta}:=\Omega \cap\left\{\left|x_{1}\right|<\delta\right\} \cap\left\{x_{2}<\gamma_{h}\left(x_{1}\right)\right\} .
$$

Singularities at $h=0$ correspond to the second term in the definition of $\varphi_{h}$.

It is straightforward that $w_{h}$ satisfies (4.8). As $\varphi_{h}$ involves the boundary function $\gamma_{h}$, the streamfunction $x_{1} \varphi_{h}$ has regularity $C^{2, \alpha}$ in the fluid domain. Moreover, $w_{h}$ is continuous across the solid boundary, so that it belongs to $C^{\infty}\left(\left(0, h_{M}\right) ; W^{1, \infty}(\Omega)\right)$. In the fluid domain, its most singular second order derivatives behave like $x_{1}^{\alpha-1}$. We deduce that $w(t, x)=w_{h(t)}(x)$ satisfies (4.2). We postpone to the appendix the proof of the following estimates:

Proposition 8 There exists $0<c<C$ such that

$$
\begin{aligned}
& \left\|w_{h}\right\|_{L^{2}(\Omega)} \leq C, \\
& c \leq h^{\frac{3 \alpha}{2(1+\alpha)}}\left\|\nabla w_{h}\right\|_{L^{2}(\Omega)} \leq C, \\
& \left\|\nabla w_{h}\right\|_{L^{\infty}\left(\Omega \backslash \Omega_{h, \delta}\right)} \leq C,
\end{aligned}
$$

and

$$
\begin{array}{r}
\sup _{x_{1} \in(-\delta, \delta)}\left|\gamma_{h}\left(x_{1}\right)\right|^{3 / 2} \\
\left.\left.\int_{-\delta}^{\delta} \int_{0}^{\gamma_{h}\left(x_{1}\right)} \gamma_{h}^{\gamma_{h}\left(x_{1}\right)} \mid \nabla w_{h}\right)\left.^{2}\left(x_{1}, x_{2}\right)\right|^{2} d x_{2}\right)\left.^{1 / 2} w_{h}(x)\right|^{2} d x \leq C,
\end{array}
$$

Besides these estimates on $w_{h}$, the control of $n(h)$ and $R(t)$ shall involve quantities of the type

$$
\int_{F(t)}\left(\mu \Delta w_{h}-\nabla q_{h}\right) \tilde{w}
$$

where $\tilde{w} \in H_{0}^{1}(\Omega)$ is divergence free and satisfies $\left.\tilde{w}\right|_{\partial S_{h}}=e_{2}$. We prove in the appendix the following estimate

Proposition 9 There exists a pressure field $h \mapsto q_{h} \in C^{\infty}\left(0, h_{M} ; C^{1}(\bar{\Omega})\right)$ such that for all divergence free $\tilde{w} \in H_{0}^{1}(\Omega)$ satisfying $\left.\tilde{w}\right|_{\partial S_{h}}=e_{2}$,

$$
\left|\int_{F(t)}\left(\mu \Delta w_{h}-\nabla q_{h}\right) \tilde{w}\right| \leq C\|\tilde{w}\|_{H_{0}^{1}(\Omega)} .
$$




\subsection{Proof of Proposition 7}

Thanks to the estimates of the previous section, we are able to control the functions $n(h)$ and the remainder term $R(t)$.

By integration by parts,

$$
n(h):=2 \mu \int_{\Omega} D\left(w_{h}\right): D\left(w_{h}\right)+\int_{F_{h}}\left(\mu \Delta w_{h}-\nabla q_{h}\right) w_{h} .
$$

By estimate (4.9) b, we have

$$
c h^{-\frac{3 \alpha}{2(1+\alpha)}} \leq\left\|\nabla w_{h}\right\|_{L^{2}(\Omega)} \leq C h^{-\frac{3 \alpha}{2(1+\alpha)}},
$$

and Proposition 9 leads to

$$
\left|\int_{F(t)}\left(\mu \Delta w_{h}-\nabla q_{h}\right) w_{h}\right| \leq C\left\|\nabla w_{h}\right\|_{L^{2}(\Omega)} \leq C_{\varepsilon}+\varepsilon\left\|\nabla w_{h}\right\|_{L^{2}(\Omega)}^{2} .
$$

Combining these last two inequalities yields point i) of Proposition 7

To establish point ii), we need to control each term in the remainder. Still using bound (4.9), we have

$$
\left|\int_{F_{h(t)}}\left(\mu \Delta w_{h(t)}-\nabla q_{h(t)}\right) v\right| \leq C\|v(t)\|_{H_{0}^{1}(\Omega)} .
$$

Integration from 0 to $t$ and Cauchy-Schwarz inequality lead to

$$
\int_{0}^{t}\left|\int_{F(s)}(\mu \Delta w(s, \cdot)-\nabla q(s, \cdot)) v(s, \cdot)\right| \leq C\|v\|_{L^{2}\left(0, t ; H^{1}(\Omega)\right)} \sqrt{t} \leq C\left(\left\|u_{0}\right\|_{L^{2}(\Omega)}\right) \sqrt{t} .
$$

We also get

$$
\begin{aligned}
\left|\int_{\Omega} \rho(0) v_{0} \cdot w(0)-\int_{\Omega} \rho(t) v(t) \cdot w(t)\right| & \leq C\|v\|_{L^{\infty}\left(0, t ; L^{2}(\Omega)\right)} \sup _{h \in\left(0, h_{M}\right)}\left\|w_{h}(t)\right\|_{L^{2}(\Omega)} \\
& \leq C\left(\left\|u_{0}\right\|_{L^{2}(\Omega)}\right)
\end{aligned}
$$

To deal with the term involving $\partial_{t} w$, we shall use the following general bound: for any $h \in\left(0, h_{M}\right)$ and any $(\rho, v) \in L^{\infty}(\Omega) \times H_{0}^{1}(\Omega)$ we have, for any $\tilde{w} \in H_{0}^{1}(\Omega)$ :

$$
\begin{aligned}
\left|\int_{\Omega} \rho v \cdot \tilde{w}\right| \leqslant C\|\rho\|_{L^{\infty}(\Omega)}\|\nabla v\|_{L^{2}(\Omega)} & \left(\|\tilde{w}\|_{L^{2}\left(\Omega \backslash \Omega_{h, \delta}\right)}\right. \\
& \left.+\left(\int_{-\delta}^{\delta} \int_{0}^{\gamma_{h}\left(x_{1}\right)}\left|\gamma_{h}\left(x_{1}\right)\right|^{2}|\tilde{w}(x)|^{2} \mathrm{~d} x\right)^{1 / 2}\right),
\end{aligned}
$$

This is a simple consequence of Cauchy-Schwarz and Hardy inequalities, and its proof is therefore left to the reader. Note that $\partial_{t} w=\dot{h}(t) \partial_{h} w_{h(t)}$. The previous formula yields

$$
\begin{aligned}
& \left|\int_{0}^{t} \int_{\Omega} \rho v \cdot \partial_{t} w\right| \leq C \sup _{\left[0, T_{*}\right)}|\dot{h}| \int_{0}^{t}\|\nabla v(s)\|_{L^{2}(\Omega)}\left(\left\|\partial_{h} w_{h(s)}\right\|_{L^{2}\left(\Omega \backslash \Omega_{h(s), \delta}\right)}\right. \\
& \left.+\left(\int_{-\delta}^{\delta} \int_{0}^{\gamma_{h(s)}\left(x_{1}\right)}\left|\gamma_{h(s)}\left(x_{1}\right)\right|^{2}\left|\partial_{h} w_{h(s)}\right|^{2} d x\right)^{1 / 2}\right) d s \leq C\left(\left\|u_{0}\right\|_{L^{2}(\Omega)}\right) \sqrt{t}
\end{aligned}
$$


where the last inequality involves (4.10) $\mathrm{b}$. Finally, to deal with the nonlinear term, we use another general formula, namely: for any $h \in\left(0, h_{M}\right)$ and any $(\rho, v) \in L^{\infty}(\Omega) \times H_{0}^{1}(\Omega)$ we have, for any $\tilde{w} \in H_{0}^{1}(\Omega)$ :

$$
\begin{aligned}
\left|\int_{\Omega} \rho v \otimes v: D(\tilde{w})\right| \leqslant & C\|\rho\|_{L^{\infty}(\Omega)}\|\nabla v\|_{L^{2}(\Omega)}^{2}\left(\|D(\tilde{w})\|_{L^{\infty}\left(\Omega \backslash \Omega_{h, \delta}\right)}\right. \\
& \left.+\sup _{x_{1} \in(-\delta, \delta)}\left(\left|\gamma_{h}\left(x_{1}\right)\right|^{\frac{3}{2}}\left(\int_{0}^{\gamma_{h}\left(x_{1}\right)}|\nabla \tilde{w}(x)|^{2} \mathrm{~d} x_{1}\right)^{\frac{1}{2}}\right)\right) .
\end{aligned}
$$

This formula follows from Cauchy-Schwarz inequality together with a refined Poincaré's inequality. We refer to lemma 12 in [20] for all necessary details. We infer from this bound and (4.10) that

$$
\int_{0}^{t} \int_{\Omega} \rho v \otimes v: D(w) \leq C\left(\left\|u_{0}\right\|_{L^{2}(\Omega)}\right) .
$$

Gathering (4.11) to (4.14) gives the bound on $R(t)$.

\section{Acknowledgements}

The authors wish to thank Bertrand Maury for a useful discussion.

\section{References}

[1] S. Agmon, A. Douglis, and L. Nirenberg. Estimates near the boundary for solutions of elliptic partial differential equations satisfying general boundary conditions. I. Comm. Pure Appl. Math., 12:623-727, 1959.

[2] G. Barnocky and Robert H. Davis. The influence of pressure-dependent density and viscosity on the elastohydrodynamic collision and rebound of two spheres. J. Fluid Mech., 209:501-519, 1989.

[3] A. Benmohamed and L. Robbiano. Exposant critique de Sobolev et régularité des solutions d'équations elliptiques. Ann. Inst. H. Poincaré Anal. Non Linéaire, 8(1):103117, 1991.

[4] H. Brenner and R. G. Cox. The resistance to a particle of arbitrary shape in translational motion at small Reynolds numbers. J. Fluid Mech., 17:561-595, 1963.

[5] C. Conca, J. A. San Martín, and M. Tucsnak. Existence of solutions for the equations modelling the motion of a rigid body in a viscous fluid. Comm. Partial Differential Equations, 25(5-6):1019-1042, 2000.

[6] M.D.A. Cooley and M.E. O'Neill. On the slow motion generated in a viscous fluid by the approach of a sphere to a plane wall or stationary sphere. Mathematika, 16:37-49, 1969.

[7] Robert H. Davis, J.M. Serayssol, and E.J. Hinch. The elastohydrodynamic collision of two spheres. J. Fluid Mech., 163:479-487, 1986.

[8] Robert H. Davis, Yu Zhao, Kevin P. Galvin, and Helen J. Wilson. Solid-solid contacts due to surface roughness and their effects on suspension behaviour. R. Soc. Lond. Philos. Trans. Ser. A Math. Phys. Eng. Sci., 361(1806):871-894, 2003.

[9] W. R. Dean and M. E. O'Neill. A slow motion of viscous liquid caused by the rotation of a solid sphere. Mathematika, 10:13-24, 1963.

[10] B. Desjardins and M. J. Esteban. Existence of weak solutions for the motion of rigid bodies in a viscous fluid. Arch. Ration. Mech. Anal., 146(1):59-71, 1999. 
[11] B. Desjardins and M. J. Esteban. On weak solutions for fluid-rigid structure interaction: compressible and incompressible models. Comm. Partial Differential Equations, 25(7-8):1399-1413, 2000.

[12] R. J. DiPerna and P.-L. Lions. Ordinary differential equations, transport theory and Sobolev spaces. Invent. Math., 98(3):511-547, 1989.

[13] C. Fefferman and E. M. Stein. $H^{p}$ spaces of several variables. Acta Math., 129(34):137-193, 1972

[14] Eduard Feireisl. On the motion of rigid bodies in a viscous incompressible fluid. $J$. Evol. Equ., 3(3):419-441, 2003. Dedicated to Philippe Bénilan.

[15] M. Giaquinta and G. Modica. Nonlinear systems of the type of the stationary NavierStokes system. J. Reine Angew. Math., 330:173-214, 1982.

[16] Mariano Giaquinta. Multiple integrals in the calculus of variations and nonlinear elliptic systems, volume 105 of Annals of Mathematics Studies. Princeton University Press, Princeton, NJ, 1983.

[17] C. Grandmont and Y. Maday. Existence de solutions d'un problème de couplage fluide-structure bidimensionnel instationnarie. C. R. Acad. Sci. Paris Sér. I Math., 326(4):525-530, 1998.

[18] M. D. Gunzburger, H.-C. Lee, and G. A. Seregin. Global existence of weak solutions for viscous incompressible flows around a moving rigid body in three dimensions. $J$. Math. Fluid Mech., 2(3):219-266, 2000.

[19] T.I. Hesla. Collisions of smooth bodies in viscous fluids : A mathematical investigation. PhD thesis, University of Minnesota, revised version, October 2005.

[20] M. Hillairet. Lack of collision between solid bodies in a 2D incompressible viscous flow. Comm. Partial Differential Equations, 32(7-9):1345-1371, 2007.

[21] K.-H. Hoffmann and V.N. Starovoitov. On a motion of a solid body in a viscous fluid. Two-dimensional case. Adv. Math. Sci. Appl., 9(2):633-648, 1999.

[22] K.-H. Hoffmann and V.N. Starovoitov. Zur Bewegung einer Kugel in einer zähen Flüssigkeit. Doc. Math., 5:15-21 (electronic), 2000.

[23] G.G. Joseph, R. Zenit, M.L. Hunt, and A.M. Rosenwinkel. Particle-wall collisions in a viscous fluid. J. Fluid Mech., 433:329-346, 2001.

[24] Jean-Lin Journé. Calderón-Zygmund operators, pseudodifferential operators and the Cauchy integral of Calderón, volume 994 of Lecture Notes in Mathematics. SpringerVerlag, Berlin, 1983.

[25] Pierre-Louis Lions. Mathematical topics in fluid mechanics. Vol. 1, volume 3 of Oxford Lecture Series in Mathematics and its Applications. The Clarendon Press Oxford University Press, New York, 1996. Incompressible models, Oxford Science Publications.

[26] B. Maury. A gluey particle model. ESAIM Proceedings, 18:133-142, 2007.

[27] J.A. San Martín, V. Starovoitov, and M. Tucsnak. Global weak solutions for the two-dimensional motion of several rigid bodies in an incompressible viscous fluid. Arch. Ration. Mech. Anal., 161(2):113-147, 2002.

[28] J.R. Smart and D.T. Leighton. Measurement of the hydrodynamic surface roughness of noncolloidal spheres. Phys. Fluids, 1:52-60, 1989.

[29] Hermann Sohr. The Navier-Stokes equations. Birkhäuser Advanced Texts: Basler Lehrbücher. [Birkhäuser Advanced Texts: Basel Textbooks]. Birkhäuser Verlag, Basel, 2001. An elementary functional analytic approach. 
[30] V.N. Starovoitov. Behavior of a rigid body in an incompressible viscous fluid near a boundary. In Free boundary problems (Trento, 2002), volume 147 of Internat. Ser. Numer. Math., pages 313-327. Birkhäuser, Basel, 2004.

[31] T. Takahashi. Analysis of strong solutions for the equations modeling the motion of a rigid-fluid system in a bounded domain. Adv. Differential Equations, 8(12):1499$1532,2003$.

[32] Juan Luis Vázquez and Enrique Zuazua. Lack of collision in a simplified 1D model for fluid-solid interaction. Math. Models Methods Appl. Sci., 16(5):637-678, 2006.

[33] Helen J. Wilson and Robert H. Davis. The viscosity of a dilute suspension of rough spheres. J. Fluid Mech., 421:339-367, 2000.

\section{Appendix : Proofs of propositions 8 and 9}

In this section, we estimate the rate of divergence of various Sobolev norms of $w_{h}$ as $h$ goes to 0. As explained in section 4, $w_{h}$ is regular up to $h=0$ in $S_{h}$ and $\Omega \backslash\left(S_{h} \cup \Omega_{h, \delta}\right)$. Hence, there holds:

$$
\left\|\nabla w_{h}\right\|_{L^{\infty}\left(\Omega \backslash \Omega_{h, \delta}\right)} \leqslant C
$$

and the rate of divergence of $w_{h}$ is the one of its restriction to $\Omega_{h, \delta}$ i.e. the one of $\nabla^{\perp}\left(x_{1} \varphi_{h}\right)$ where

$$
\varphi_{h}(x)=\frac{x_{2}^{2}}{\gamma_{h}\left(x_{1}\right)}\left(3-2 \frac{x_{2}}{\gamma_{h}\left(x_{1}\right)}\right), \quad \forall x \in \Omega_{h, \delta} .
$$

Proposition 8 is then a straightforward consequence of:

Lemma 10 Given $(\alpha, p, q) \in(0, \infty)^{3}$, the quantity:

$$
\int_{-\delta}^{\delta} \frac{\left|x_{1}\right|^{p} d x_{1}}{\left(h+\left|x_{1}\right|^{1+\alpha}\right)^{q}}
$$

behaves like

$$
\begin{array}{lll}
\text { (i) } & c h^{\frac{(p+1)}{1+\alpha}-q}, & \text { if } p+1<q(1+\alpha), \\
\text { (ii) } \quad c \ln (h), & \text { if } p+1=q(1+\alpha), \\
\text { (iii) } c, & \text { if } p+1>q(1+\alpha) .
\end{array}
$$

when $h$ goes to 0 , with $c$ a constant depending only on $(\alpha, p, q)$.

The proof of this lemma as well as the induced bounds on $w_{h}$ are direct adaptation of [20, Lemma 13].

It remains to build the pressure field $q_{h}$ in order to prove proposition 9. For simplicity, we assume now $\mu=1$. With the same notations as in section 4 , we set:

$$
q_{h}(x)=\partial_{21}\left(x_{1} \varphi_{h}(x)\right)+12 \int_{0}^{x_{1}} \frac{t}{\gamma_{h}\left(x_{1}\right)^{2}} \mathrm{~d} t, \quad \forall x \in \Omega .
$$

We stress that

$$
q_{h}(x)=\partial_{21}\left(x_{1} \varphi_{h}(x)\right)-\int_{0}^{x_{1}} \partial_{222}\left(t \varphi_{h}\left(t, x_{2}\right)\right) \mathrm{d} t, \quad \forall x \in \Omega_{h, \delta} .
$$

As for $w_{h}$, this pressure field is smooth up to $h=0$ in the fluid domain outside $\Omega_{h, \delta}$. Consequently, the rate of divergence of $\Delta w_{h}-\nabla q_{h}$ is the one of its restriction to this latter domain. Standard computations lead to :

$$
\Delta w_{h}(x)-\nabla q_{h}(x)=\left(\begin{array}{c}
-2 \partial_{112}\left(x_{1} \varphi_{h}(x)\right) \\
\partial_{111}\left(x_{1} \varphi_{h}(x)\right)
\end{array}\right) \quad \forall x \in \Omega_{h, \delta} .
$$


We recall that $\nabla^{2} w_{h} \in L^{p}\left(\Omega_{h, \delta}\right)$ for $p$ sufficiently small. As $H^{1}\left(\Omega_{h, \delta}\right) \subset L^{r}\left(\Omega_{h, \delta_{0}}\right)$ for arbitrary $r<\infty$, the integral to be estimated in proposition 9 is well-defined. Up to a truncation (which leaves aside a term that is regular with respect to $h$ ), we can assume $\tilde{w}=0$ in $\left(\Omega \backslash S_{h}\right) \backslash \Omega_{h, \delta}$. A fortiori:

$$
\int_{\Omega \backslash S_{h}}\left(\Delta w_{h}-\nabla q_{h}\right) \cdot \tilde{w}=\int_{\Omega_{h, \delta}}\left(\Delta w_{h}-\nabla q_{h}\right) \cdot \tilde{w} .
$$

After an integration by parts, accounting for $\left.\tilde{w}\right|_{\partial S_{h}}=\left(0, \tilde{w}_{2}\right)$ :

$$
\int_{\Omega_{h, \delta}}\left(\Delta w_{h}-\nabla q_{h}\right) \cdot \tilde{w}=-\int_{\partial S_{h}} \partial_{11}\left(x_{1} \varphi_{h}\right) \tilde{w}_{2} n_{1} \mathrm{~d} \sigma-\int_{\Omega_{h, \delta}} \partial_{11}\left(x_{1} \varphi_{h}\right)\left(2 \partial_{2} \tilde{w}_{1}-\partial_{1} \tilde{w}_{2}\right) .
$$

Thanks to Lemma10, one can check that $\partial_{11}\left(x_{1} \varphi_{h}\right)$ is bounded uniformly in $h$ in $L^{2}\left(\Omega_{h, \delta}\right)$. then shows $\left\|\partial_{11} \varphi_{h}\right\|_{L^{2}\left(\Omega_{h, \delta}\right)}$. Moreover, the boundary term reads

$$
\left|\int_{\partial S_{h}} \partial_{11}\left(x_{1} \varphi_{h}\right) \tilde{w}_{2} n_{1} \mathrm{~d} \sigma\right| \leq\left\|\tilde{w}_{2}\right\|_{L^{\infty}\left(\partial \Omega_{h, \delta}\right)} \int_{0}^{\delta}\left|\frac{6 x_{1}\left(\gamma_{h}^{\prime}\left(x_{1}\right)\right)^{2}}{\left(\gamma_{h}\left(x_{1}\right)\right)^{2}} \frac{\gamma_{h}^{\prime}\left(x_{1}\right)}{1+\left(\gamma_{h}^{\prime}\left(x_{1}\right)\right)^{2}}\right| d x_{1}
$$

where $\left|\gamma_{h}^{\prime}\left(x_{1}\right)\right| \leqslant c\left|x_{1}\right|^{\alpha}$. So, this boundary term is again uniformly bounded by Lemma 10. This ends the proof of proposition 9 . 